\title{
Squeezed excitation in cavity QED: Experiment and theory
}

\author{
Q. A. Turchette, N. Ph. Georgiades, C. J. Hood, and H. J. Kimble \\ Norman Bridge Laboratory of Physics, California Institute of Technology, Pasadena, California 91125
}

\author{
A. S. Parkins \\ University of Auckland, Auckland, New Zealand
}

(Received 12 January 1998)

\begin{abstract}
One of the canonical questions in quantum optics is the nature of the radiative properties of an atom when the normal vacuum fluctuations of the electromagnetic reservoir are replaced by the asymmetric, reduced fluctuations of a squeezed vacuum. While the basic radiative linewidth-narrowing effect has been known for over a decade [C. W. Gardiner, Phys. Rev. Lett. 56, 1917 (1986)], experimental realizations with operationally definable definitive manifestations of the quantum nature of the squeezed reservoir have been largely lacking from subsequent investigations. This paper presents measurements on an experimentally realized atomsqueezed-light system, in which the squeezed-light output of a subthreshold optical parametric oscillator illuminates an atom strongly coupled to a high-finesse optical resonator. Transmission of a weak probe field incident on the atom-cavity system is investigated both theoretically and experimentally. Alteration of the transmitted probe spectrum has been observed, as has a transmission modulation that depends on the phase of the squeezed field relative to a saturating coherent field (displaced squeezing). In certain parameter regimes, properties unique to the quantum nature of the squeezed light have been identified in the theoretical treatment, but complications in the experiment prevent their unequivocal measure. It is found that the observed effects of the squeezed light are dramatically reduced relative to the predictions of an idealized theory. This is quantitatively attributed to the effects of atomic beam fluctuations and a simple modeling of the atomic beam as an additional loss mechanism in the theory leads to reasonable agreement with the data. [S1050-2947(98)05510-3]

PACS number(s): 42.50.Dv, 42.50.Ct, 32.80.-t
\end{abstract}

\section{INTRODUCTION}

It has been known for quite some time that squeezed vacuum can have significant effects on the radiative properties of atoms [1-3]. Demonstrating this in the laboratory has proved to be a demanding and difficult task, with to date only one experiment reported [4]. Given that realizable experimental systems are so scarce, so too are operational criteria for identifying what is and what is not quantum mechanical about the atom-squeezed-field interaction.

In addition to a general lack of squeezed light sources coincident with convenient atomic transitions, the dearth of experimental realizations can be attributed to the difficulty of efficient coupling of squeezed optical "beams" to the dipole radiation pattern of an atom and to the efficient collection of the scattered radiation from the interaction. To quantify this situation, consider the prediction due to Gardiner [1] of reductions and enhancements in the spontaneous decay of the components of the atomic polarization proportional to the variances $\Delta X_{\mp}^{2}$ of the "quiet" and "noisy" quadratures of the squeezed vacuum. In a realistic experiment, the decay rates $\beta_{ \pm}$of an atom in squeezed vacuum can be altered from the free space value $\gamma_{\perp}$ only according to $\beta_{ \pm}=\gamma_{\perp}[(1$ $\left.\left.-\eta_{f}\right)+\eta_{f} \Delta X_{ \pm}^{2}\right]$, where $\eta_{f}$ is a measure of the efficiency with which the squeezed field is coupled to the atom. For an atom in free space illuminated by Gaussian beams of squeezed light, the overlap of the incident field distribution with the dipole radiation pattern is typically very small $\left(\eta_{f}\right.$ $\sim 10^{-4}$ ). The atom-squeezed-light coupling is then so weak that any effect of the quiet quadrature $\Delta X_{-}$of the squeezed field is masked by the "normal" vacuum fluctuations in modes not occupied by the squeezed field, but with which the atom nonetheless interacts.

In principle, this weak-coupling situation can be completely different if the atom is placed in a cavity in such a way that interaction with a single electromagnetic mode of the cavity dominates the evolution of the atomic degrees of freedom, in which case only this single mode need be squeezed to have a dramatic effect on the atom. For an atomcavity system in the "one-dimensional" atom (1D atom) regime [5] the basic physical effect of reductions and enhancements in atomic decay rates remains very much the same, since the evolution time scales of the atom and the cavity are well separated, and the problem of efficient coupling of the squeezed light to the atom is reduced to that of effective mode matching of a beam of squeezed light to the cavity. The effect of the squeezed vacuum on the 1D atom is reflected in an inhibition of the cavity-enhanced spontaneous emission component of the 1D atom spectrum. With an atom-field cooperativity parameter $C_{1}$, the radiative rate alterations can be described by $\beta_{ \pm}^{c}=\gamma_{\perp}\left[1+2 C_{1} \Delta X_{ \pm}\right]$, where for the case of perfect squeezing (with $\Delta X_{-} \rightarrow 0$ ) $\beta_{-}^{c} \rightarrow \gamma_{\perp}$, representing a reduction in the rate of decay described by the atomlike eigenvalue of the coupled atom-cavity system. Note that an increase in coupling efficiency $\eta_{f}$ could also be achieved by using a large solid-angle cavity $[6,7]$.

\section{MOTIVATION AND BACKGROUND}

\section{A. Historical interest}

Since the first few "seminal" works on the subject by Gardiner [1] and Milburn [2,3], there has been considerable 
theoretical interest on the interaction of squeezed light and atoms. To provide a backdrop for the present work, we present a short compendium of the topics covered by diverse theoretical treatments. Extension of the early work on the basic radiative properties of atoms in ideal squeezed light has continued unabated $[8-12,1,13-20]$ with some consideration of atoms in finite bandwidth squeezing [21-23] and much attention to resonance fluorescence of atoms in squeezed vacuum [24-36]. Optical bistability in squeezed vacuum has been considered [37], as have optical pumping with squeezed light [38] and photon echoes and revivals $[39,40]$. There was intense interest in lasers with squeezed pump fields or squeezed reservoirs [41-47], gain without inversion $[48,49]$, and electromagnetically induced transparency in squeezed light [50]. Squeezed light interacting with atoms in cavities has been given significant coverage [51-57]. Two proposals in the bad cavity limit are particularly relevant to our work $[58,59]$ and a proposal in the strong-coupling limit has great promise [60]. Opportunities for laser cooling in squeezed vacuum [61-64] have been explored. The correlated pairs of photons in squeezed light have been considered in conjunction with three-level atoms and two-photon effects [65-76]. There are at least two papers related to the consequences of the phase of the squeezing [77,78]. Interesting ideas involving cooperative effects with multiple atoms in squeezed vacuum [79-84] have been noted. A review covering many of the above topics can be found in Ref. [85].

This abridged collection of theoretical work is well ahead of a limited number of experiments, which in fact number only two: one is reported here and the other, also from our group, was published earlier in Ref. [4]. The goal of these experiments is to observe effects that are purely nonclassical in the sense that the properties of the squeezed-light-atom system to be observed must be the unique result of the nonclassical nature of the electromagnetic field used in the experiment. That is, we require that for all classical states of light, these effects cease to exist. Clearly this imposes several theoretical as well as experimental challenges. In particular, we point out that for a certain class of states, the so-called classical squeezed states (see Sec. I C), many of the qualitative features are very similar to features present with quantum squeezed light and only fine tuning of the parameters could in principle convince one of the intrinsic quantum nature of the observations. In the work of Ref. [4], this difficulty was bypassed by identifying an effect whose qualitative feature was unique to quantum squeezing.

In our current work, however, we do not have the luxury of unique qualitative effects and in fact the features of the effects we report could for the most part be reproduced by the use of classical squeezed states. Despite these difficulties, we note that our experiment is one of only two in existence in which radiative alterations in the interaction of squeezed light with atoms have been observed. In addition, we note that when certain aspects of the experiment are improved, then careful quantitative measurements in relatively unconstrained regions of the parameter space will reveal unique effects that could not be observed with any other kind of classical radiation. We also note that, to our knowledge, our experiments represent the only realization of the coupling of two distinct complex quantum systems and certainly repre- sent the only attempt to excite a cavity QED system with nonclassical light.

In a broader context, our particular realization of the squeezed-light-atom-cavity is an instance of a unidirectional cascaded quantum system. In such a system, the output field of one quantum system drives, or provides the input field for another quantum system. It is a scenario that offers a simple and elegant theoretical treatment $[86,87]$, but which has not to any significant extent been realized in the laboratory.

The paper is arranged as follows. In the rest of this section we give brief descriptions of the relevant properties of the 1D atom and squeezed light and then present a simple recapitulation of the well-known ideas in the interaction of squeezed light with atoms in free space and in cavities. With the description of the experimental system presented in Sec. II, it becomes quickly apparent that the simple theory of Sec. I leaves out an important aspect of a realization of the atomcavity-squeezing system: getting the squeezed light into the cavity to interact with the atom. Having addressed this issue, we move on to the measurements themselves and present data of two types for a variety of parameters: direct spectroscopy of the 1D atom-squeezing system and a phasesensitive modulation technique. In Sec. III we present a recently derived (somewhat simplified) analytic theory of our system that is capable of delineating those regions of parameter space that are of primary import, that is, where the squeezing theory differs from one with a classical asymmetric noise field. Finally, in Sec. IV we present a detailed comparison of the theory of Sec. III applied to the data of Sec. II (the reader not interested in the details of the experiment or theory could skip directly to Sec. IV and Fig. 17 for a summary of the whole effort). The agreement is quite good, albeit in a regime of the theory that is not clearly able to distinguish purely quantum-mechanical effects from classical ones. This is due to complications of the experiment resulting from use of an atomic beam, which gives rise to additional loss mechanisms that reduce the effects of the squeezed field interaction with single atoms. We have quantitative details that indicate that this nonideal system is the root of difficulties in the experiment.

\section{B. Brief discussion of the properties of the 1D atom}

The one-dimensional atom consists of an atom strongly coupled to a single electromagnetic mode of a high-finesse optical cavity. Details of previous measurements on the system are described in Ref. [5], while a detailed theoretical discussion of cavity QED in the bad-cavity limit can be found in Ref. [88]. In this paper we are most interested in two particular properties of the 1D atom: its linewidth and its saturation behavior. The linewidth of the $1 \mathrm{D}$ atom is described by the width of the central absorption feature in the transmission spectrum of a weak-probe beam, given by

$$
\gamma_{\perp}^{c}=\gamma_{\perp}\left(1+2 N_{e} C_{1}\right),
$$

where $\gamma_{\perp}$ is the decay rate of the atomic polarization to modes other than the privileged cavity mode (here $\gamma_{\perp}$ is approximately equal to the free space decay rate), $N_{e}$ is the effective number of atoms in the cavity $\left(N_{e} \leqslant 1\right.$ for all of our measurements), and $C_{1}$ is the single-atom cooperativity parameter, which is the part of the decay governed by the cav- 
ity. For our system with dipole coupling rate $g_{0}$ and cavity decay rate $\kappa$ [half-width at half maximum (HWHM)],

$$
C_{1}=\frac{g_{0}^{2}}{2 \kappa \gamma_{\perp}} \text {. }
$$

The effective number of atoms for a sample of $N_{s}$ atoms distributed within the cavity mode volume is given by

$$
N_{e}=\sum_{l=1}^{N_{s}}\left|\psi\left(\vec{r}_{l}\right)\right|^{2}
$$

where $\psi\left(\vec{r}_{l}\right)=\cos k z \exp \left[-\left(x^{2}+y^{2}\right) / w_{0}^{2}\right]$ is the mode function of our Gaussian standing-wave cavity, with $k=2 \pi / \lambda$ the wave number of the two-state atomic transition. The probe transmission spectrum for the system is thus a broad, essentially unaltered Lorentzian of width $\kappa$ due to the cavity, with an atomic absorption "dip" in its center, the half-width of which is given by Eq. (1). In addition, the depth of the dip on resonance $\left(\omega_{A}=\omega_{c}=\omega_{p}\right)$ is given by

$$
T=T_{0} \frac{1}{\left(1+2 N_{e} C_{1}\right)^{2}},
$$

where $T_{0}$ is the empty-cavity peak transmission.

The second property of interest is the saturation. This is essentially the behavior of the probe transmission as the (fixed-frequency) probe is itself increased in strength or as another coherent field drives the system with increasing strength. (It is the latter that we use here.) The qualitative saturation of the 1D atom for the particular parameter regime considered here is fairly well described by the state equation for optical bistability [89]. In particular, the onset of atomic saturation for a resonant field [the point at which $T$ $\left.\rightarrow 2 T_{0}\left(1+2 N_{e} C_{1}\right)^{-2}\right]$ occurs at approximately the saturation photon number $m_{0}=2 \gamma_{\perp} \gamma_{\|} / 3 g_{0}^{2}$ familiar from the bistability literature. The measured saturation of the $1 \mathrm{D}$ atom is presented in Ref. [90].

\section{Brief discussion of the properties of squeezed light}

Following the standard description for the squeezed output from a subthreshold optical parametric oscillator (OPO) of Collet and Louden [91], we begin by defining the parameter $x$ to be

$$
x \equiv \frac{\varepsilon}{\kappa_{s} / 2},
$$

where $\varepsilon$ is the strength of the parametric driving rate of the OPO and $\kappa_{s}$ the full width at half maximum (FWHM) of the cold (i.e., no pump) OPO cavity. This parameter $x$ is to be understood as the pumping parameter with a range between 0 and 1 with 0 corresponding to no pumping and 1 being the threshold beyond which the OPO starts to "lase." The degree of squeezing and other properties of the electromagnetic field at the output of the OPO are then expressed in terms of $x$. Of particular relevance to our experiment is the phase sensitive gain $G_{ \pm}$that can be measured readily by observing the phase sensitive amplification $G_{+}$or deamplification $G_{-}$ of a small injected coherent signal into the OPO. $G_{ \pm}$and $x$ are related via [92]

$$
G_{ \pm}=\frac{1}{(1 \mp x)^{2}} .
$$

A convenient parametrization of squeezed vacuum that has been widely adopted in theoretical studies uses the quantities $N$ and $M$, defined through the stationary two-time correlation functions for the output field from the squeezing source,

$$
\begin{aligned}
& \left\langle a_{\text {out }}^{\dagger}(t+\tau) a_{\text {out }}(t)\right\rangle=N f(|\tau|), \\
& \left\langle a_{\text {out }}(t+\tau) a_{\text {out }}(t)\right\rangle=M h(|\tau|),
\end{aligned}
$$

where $a_{\text {out }}(t)$ and $a_{\text {out }}^{\dagger}(t)$ denote the output field annihilation and creation operators and $f(|\tau|)$ and $h(|\tau|)$ are certain functions of $\tau[f(|\tau|), h(|\tau|) \rightarrow \delta(\tau)$ in the limit of broadband squeezing]. For a minimum-uncertainty squeezed state

$$
|M|=\sqrt{N(N+1)} .
$$

$M$ and $N$ are a complete specification of the degree of squeezing, as is $N$ alone for a minimum-uncertainty state.

These parameters can also be expressed in terms of the pumping parameter $x$. Via the relation of $x$ to the measured quantity $G_{ \pm}$one may eliminate $x$ in favor of $G_{ \pm}$to arrive at

$$
N=\frac{4 G_{+}\left(\sqrt{G_{+}}-1\right)^{2}}{\left(2 \sqrt{G_{+}}-1\right)^{2}}
$$

and

$$
M=\frac{2 \sqrt{G_{+}}\left(\sqrt{G_{+}}-1\right)\left(2 G_{+}-2 \sqrt{G_{+}}+1\right)}{\left(2 \sqrt{G_{+}}-1\right)^{2}} .
$$

Furthermore, we define a measure of the bandwidth of the squeezing as a quantity $b_{ \pm}$, which depends on the phase of the squeezing. Also we note that $b_{ \pm}$are functions of the pumping parameter, with $\left(b_{+}, b_{-}\right) \rightarrow\left(\kappa_{s} / 2, \kappa_{s} / 2\right)$ for the case of very weak pumping $(x \rightarrow 0)$, while in the opposite extreme of very strong pumping approaching threshold ( $x$ $\rightarrow 1),\left(b_{+}, b_{-}\right) \rightarrow\left(\kappa_{s}, 0\right)$. Again by eliminating the dependence of the bandwidth parameters $b_{ \pm}$on the pumping parameter $x$ in favor to the measured quantity $G_{+}$, one arrives at

$$
b_{+}=\frac{\kappa_{s}}{2}(1+x)=\kappa_{s}\left(1-\frac{1}{2 \sqrt{G_{+}}}\right)
$$

and

$$
b_{-}=\frac{\kappa_{s}}{2}(1-x)=\frac{\kappa_{s}}{2 \sqrt{G_{+}}} .
$$

Now, let us turn to the quantum nature of the output of the OPO and define what states are to be considered as quantum. It can be shown that the spectrum of squeezing as defined, for example, in Ref. [93] "dips" below the vacuum-state 


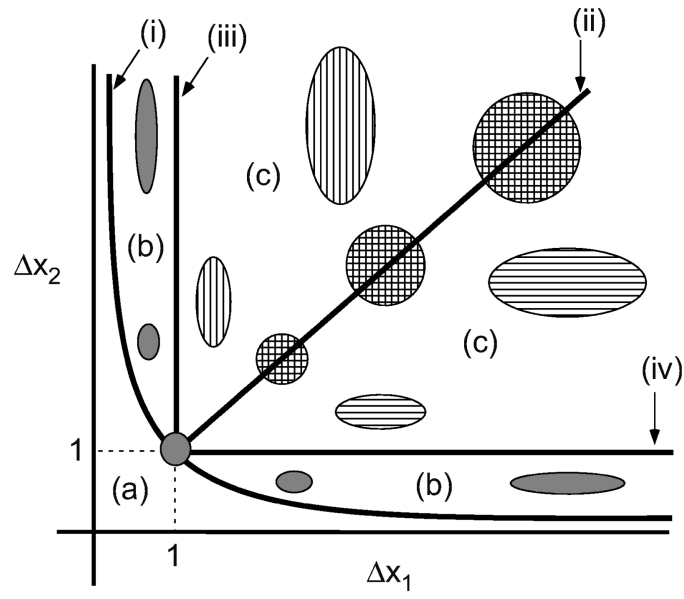

FIG. 1. Quadrature space of the squeezed states of the electromagnetic field. Curve $(i)$ indicates the minimum uncertainty relation $\Delta x_{1} \Delta x_{2}=1$, curve (ii) is for the case that $\Delta x_{1}=\Delta x_{2}$, and curves (iii) and (iv) are for $\Delta x_{1}=1$ and $\Delta x_{2}=1$, respectively. On this space, states in region $(a)$ are forbidden by Heisenberg's uncertainty relation for the quadratures of the quantized electromagnetic field, namely, $\Delta x_{1} \Delta x_{2} \geqslant 1$. States in the region $(b)$ are quantum states characterized by the fact that one of the two quadratures has magnitude less than 1 and correspond to the cases where $N<M$ $\leqslant \sqrt{N(N+1)}$. States in region $(c)$ [including the locus of curve $(i i)$ ] are classical states with both quadratures bigger than 1 and correspond to the case that $M<N$. The special state for which $\Delta x_{1}$ $=\Delta x_{2}=1$ is the electromagnetic vacuum for which $M=N=0$, while all states with $\Delta x_{1}=\Delta x_{2}>1$ are thermal states for which $N$ $>0$ and $M=0$.

level if and only if $M>N$. This criterion will be the benchmark of what we will henceforth refer to as quantum states of light. In particular, for minimum-uncertainty states, $M$ and $N$ have an exact relation given by

$$
M=\sqrt{N(N+1)} .
$$

This constitutes the maximum nonclassical behavior allowed; however, for all states with $N<M \leqslant \sqrt{N(N+1)}$ the squeezing is still quantum in the sense that homodyne detection of the field will reveal noise suppression below the shotnoise level. In the presence of nonperfect coupling efficiency $\eta$ between the OPO and detection sites (where detection in the context of the present experiment would be the interaction region of the squeezed light with the atoms), $N \rightarrow \eta N$ $=N^{\prime}, M \rightarrow \eta M=M^{\prime}$, and the above criterion is modified to $N^{\prime}<M^{\prime}<\eta \sqrt{N(N+1)}$. Note that although $M$ is decreased, its quantum nature persists.

Turning now to "all other' states of light, i.e., all classical states of light, the above criterion implies that classicality onsets for states with $M \leqslant N$. This is quite different from and in fact much more stringent than the oft-used comparison of theories with $M=0$ and $M=\sqrt{N(N+1)}$. By investigating these two extremes only, one ignores the very large set of classical squeezed states for which $0<M<N$. Note that the class of states with $M=0, N>0$ are the thermal states while the unique $M=0, N=0$ state is the vacuum. In a cartoonlike graph of the two orthogonal quadratures of the electromagnetic field, we show in Fig. 1 the various regimes discussed above. Finally, we note that to generate displaced squeezed states, which we have used for a large part of our current work, we have mixed on a 99-1 beam splitter the output of the OPO with a phase-coherent laser beam of controllable amplitude.

\section{Simple theory of squeezing and atoms}

\section{Free-space atom and squeezing}

It is useful to review the basic results of the simplified free-atom case, in which the squeezed field precisely matches the dipole-radiation pattern of the atom. An atom decaying to such a squeezed-vacuum reservoir covering a region of the electromagnetic spectrum much larger than the atomic bandwidth $\left(\kappa_{s} \gg \gamma_{\|}\right)$obeys the following optical Bloch equations (OBE's) for the mean dipoles and inversion [1]:

$$
\begin{aligned}
& \left\langle\dot{\sigma}_{x}\right\rangle=-\gamma_{\perp}(N-M+1 / 2)\left\langle\sigma_{x}\right\rangle, \\
& \left\langle\dot{\sigma}_{y}\right\rangle=-\gamma_{\perp}(N+M+1 / 2)\left\langle\sigma_{y}\right\rangle, \\
& \left\langle\dot{\sigma}_{z}\right\rangle=-\gamma_{\perp}(2 N+1)\left\langle\sigma_{z}\right\rangle-\gamma_{\perp},
\end{aligned}
$$

where $\sigma_{x}, \sigma_{y}, \sigma_{z}$ are the atomic Pauli operators. The spectrum of light emitted from the atom illuminated by squeezed vacuum consists of two parts: a flat wide component of width $\gamma(N+M+1 / 2)$ associated with $\Delta X_{+}$and a component of arbitrarily narrow width $\gamma(N-M+1 / 2)$ associated with $\Delta X_{-}$. The narrowing of the $\Delta X_{-}$component is a property that is unique to quantum light, in that it only occurs when $M>N$. It is rare, however, that the quadrature decay constants are directly measured. It is more common to measure the spectrum of fluorescence, which depends on the way each of the three $\sigma$ 's decays. In this case, it turns out that the spectrum of fluorescence is still a useful indicator of the quantum nature of the interaction since the narrow component eventually dominates the spectrum giving rise to a linewidth that is indeed below the natural linewidth of the atom. This is a direct result of the reduced fluctuations in the quiet quadrature of the squeezed vacuum and is known as inhibition of atomic phase decays [1,94]. In fact, Gardiner [1] considered the atom as a detector of squeezed light, which responds with a narrowed spectrum of fluorescence, and also pointed out the difficulties encountered when the overlap of the atomic dipole and the squeezed reservoir is poor.

\section{Atom in cavity and squeezing}

In this section we will lay the foundations of the theoretical treatment of an atom in a cavity in which the cavity mode is excited with a squeezed field. The details will be left to Sec. III. We consider the squeezing-atom-cavity system only in the 1D atom limit in which the separation of time scales between the atom (enhanced) decay and the cavity decay allows the atom to be considered as a (modified) independent entity. In general, for both broadband and narrowband squeezing, it is possible to derive relatively simple Bloch equations that should describe the atomic dynamics with reasonable accuracy. 
Assume to start that the system composed of atom plus cavity plus squeezing obeys OBE's of the form [53]

$$
\begin{gathered}
\left\langle\dot{\sigma}_{x}\right\rangle=-\gamma_{x}\left\langle\sigma_{x}\right\rangle, \\
\left\langle\dot{\sigma}_{y}\right\rangle=-\gamma_{y}\left\langle\sigma_{y}\right\rangle+\Omega_{z}\left\langle\sigma_{z}\right\rangle, \\
\left\langle\dot{\sigma}_{z}\right\rangle=-\gamma_{z}\left\langle\sigma_{z}\right\rangle-\frac{\gamma}{2}\left(1+2 C_{1}\right)-\Omega_{y}\left\langle\sigma_{y}\right\rangle,
\end{gathered}
$$

where $\gamma_{x, y, z}$ are sensitive to the phase of the squeezing and also depend on other parameters of the squeezing. $\Omega_{y, z}$ represent the effect of a coherent drive field that defines the reference for the phase of the squeezing. We can consider either the case of squeezed vacuum $\left(\Omega_{y, z}=0\right)$ or a displaced squeezed field, in which $\Omega_{y, z}$ will be related to the amplitude of the displacing coherent field $\Omega$ in some way that depends on the actual buildup of photons in the cavity, which in turn depends on the decay of the atoms and hence ultimately on the parameters of the squeezing. This approach will be justified and discussed in much more detail in Sec. III. We will now set $\Omega=0$ and consider only a broadband squeezing model originally formulated by Rice and Pedrotti [58].

Broadband squeezed light has a spectrum of squeezing that is broader than any of the atom-cavity rates: $\kappa_{s}$ $\gg g, \kappa, \gamma$, with $\kappa_{s} \rightarrow \infty$ defining infinite bandwidth squeezing. In the infinite bandwidth squeezed vacuum case (with $\Omega$ $=0)$, Rice and Pedrotti [58] show that

$$
\begin{gathered}
\gamma_{x}=\gamma_{\perp}\left[1+2 C_{1}(1+2 N-2 M)\right], \\
\gamma_{y}=\gamma_{\perp}\left[1+2 C_{1}(1+2 N+2 M)\right], \\
\gamma_{z}=\gamma_{\|}\left[1+2 C_{1}+4 C_{1} N\right] .
\end{gathered}
$$

The phase of the squeezing is taken to line up with one of the quadrature decay channels of the atom. (If we take the opposite phase $\gamma_{x}$ and $\gamma_{y}$ change roles.) In the limit of strong squeezing, from Eq. (9) $M \approx N+1 / 2$ and

$$
\begin{gathered}
\gamma_{x}=\gamma_{\perp}, \\
\gamma_{y}=\gamma_{\perp}\left[1+4 C_{1}+8 C_{1} N\right], \\
\gamma_{z}=\gamma_{\|}\left[1+2 C_{1}+4 C_{1} N\right] .
\end{gathered}
$$

Thus there is an inhibition of the cavity-enhanced spontaneous emission [compare Eq. (24) with $\gamma_{\perp}\left(1+2 C_{1}\right)$ from Eq. (1)]. Strictly speaking, this interpretation and derivation are valid only with an infinitely wide cavity (for nonzero $\left.g_{0}, \gamma_{\perp}\right)$, in the sense of the true "bad-cavity" limit [88].

Rice and Pedrotti [58] focused their attention on the spectrum of fluorescence out the side of the cavity, but a simple extension of their work (or a special case of our own from Sec. III) can reveal the transmitted probe spectrum, which is the quantity measured in our experiments, and the measurement of choice since it utilizes maximally the convenient one dimensionality of the atom-cavity system. This quantity $\left[\left|A_{p}(\nu)\right|^{2}\right.$ from Eq. (39)] is shown in Fig. 2 for several values of $N$ for the broadband case. Note that the "dip" associated with the 1D atom gets both shallower (the squeezed vacuum carries photons that saturate the atom) and narrower.

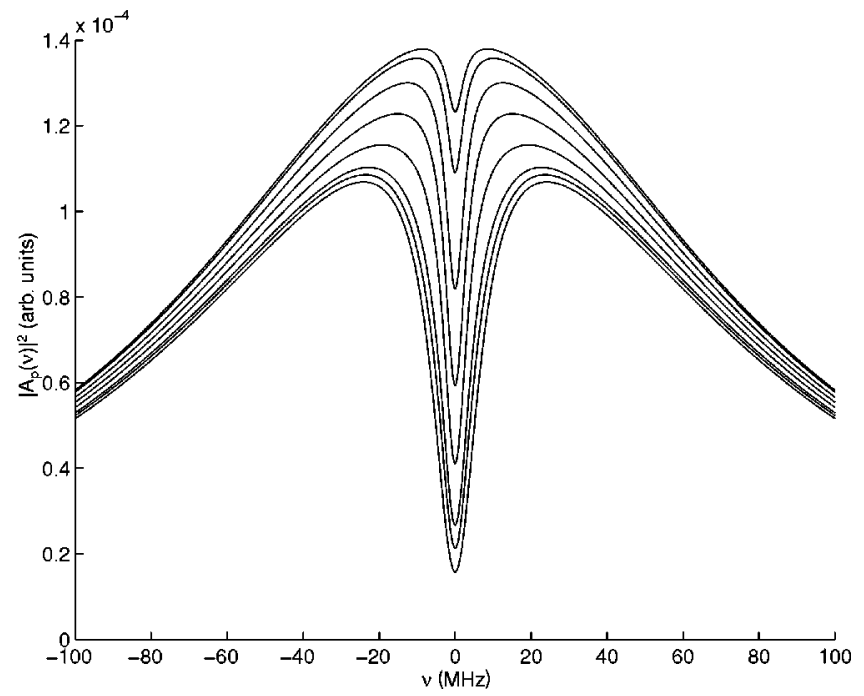

FIG. 2. The effect of broadband squeezed vacuum on the transmission spectrum of an atom-cavity system, for various degrees of squeezing. From bottom to top, $N=0,0.1,0.2,0.5,1,2,5$, and 10 . Note that this calculation is done in the strict bad cavity limit in which there is no atom-inhibited cavity decay. $C_{1}=1$ and $\left(g_{0}, \gamma_{\perp}, \kappa\right) / 2 \pi=(20,2.5,80) \mathrm{MHz}$.

Already it is seen that the simple linewidth narrowing described by Eq. (21) is not immediately obvious from the probe transmission spectrum since the depth and the width of the dip change together.

A primary aim of the current research is to determine to what extent the measurable features of the system (such as the alteration of the transmission dip of Fig. 2) are uniquely due to the quantum character of the squeezed light. There is no question that the reduced decay constant $\gamma_{x}$ is only reduced if the light is quantum squeezed $(M>N)$, but the probe transmission spectrum and the spectrum of fluorescence depend in complicated ways not only on $\gamma_{x}$, but also on $\gamma_{y}$ and $\gamma_{z}$, which are not reduced relative to their vacuum values. It thus remains to determine if the measurable quantities are also robust indicators of the nature of the light. Figure 3 addresses this issue. We show the "width" of the spectrum of fluorescence [out the sides of the cavity, taken

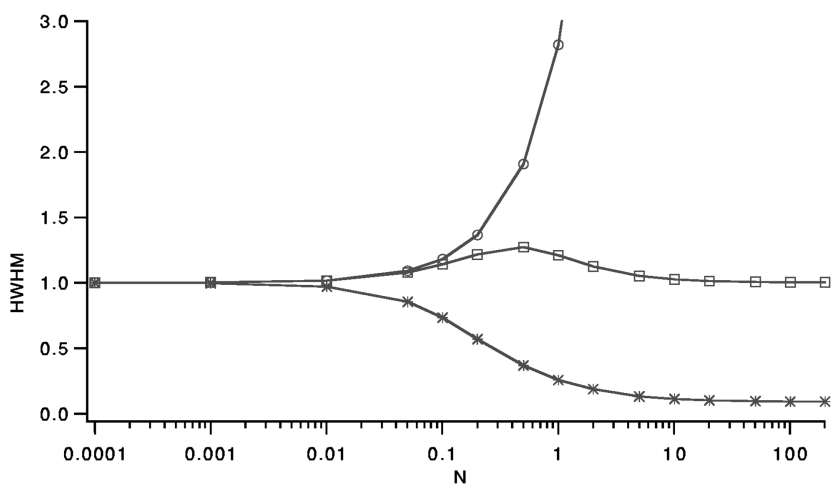

FIG. 3. Width of the spectrum of fluorescence for various kinds of light, normalized by the cavity-enhanced linewidth $(\gamma / 2)(1$ $+2 C$ ). Quantum squeezing, *; classical squeezing, $\square$; thermal light, $\bigcirc$. These results are from a simple application of the formalism of Ref. [58]; see the text. 
from plots of Eq. (16) from Ref. [58]] for excitation with light of various statistics, characterized by the parameters $M$ and $N$ of Eqs. (8) and (7). A thermal field has $M=0$, while classical squeezing has asymmetric fluctuations in which neither quadrature goes below the vacuum level, with $M=N$. Neither of these types of light shows the same linewidth narrowing as the squeezed light. Note that a strong coherent field also has an effect on the spectrum of transmitted light that is similar (at least superficially) to that shown in Fig. 2 (this can be seen, for example, in Fig. 7 of Ref. [5]). We have also determined that a coherent field will have a significantly different behavior from a squeezed field.

\section{EXPERIMENT}

The experiment uses two complete and independent laboratories: Laboratory 1 contains the apparatus associated with the $1 \mathrm{D}$ atom and laboratory 2 the apparatus to generate the resonant squeezed light. Recent experimental results from laboratory 1 can be found in Refs. [5,90], with a detailed discussion of the apparatus and procedure in Ref. [5]. For laboratory 2, recent experimental results are reported in Refs. [4,95-97] with detailed discussions of the generation of frequency-tunable squeezed light in Refs. [95-97]. Here we will only explain each laboratory in general terms focusing on techniques that are new to this experiment. Note that one of the challenges of the experiment has been the interfacing of the two laboratories, where the squeezed light generated in laboratory 2 has to be transported efficiently over a distance of 5-7 $\mathrm{m}$ and through a separating wall to the site of the cavity QED setup in laboratory 1, and then efficiently mode matched to the high-finesse cavity of the 1D atom. As the losses in the path of the squeezed light must be kept to an absolute minimum and the polarization rigorously preserved, optical fibers are unacceptable, so we simply drilled a hole in the wall between the two laboratories and sent the squeezed light from one optical table to the other, completely independent table.

\section{A. Laboratory 1: 1D atom}

\section{Description and parameters}

As indicated in Fig. 4, the cavity is a Fabry-Pérot of length $l=50 \mu \mathrm{m}$ with mirror $M_{1}$ with power transmission $\delta_{1} \approx 1 \times 10^{-6}$ and $M_{2}$ with transmission $\delta_{2} \approx 322 \times 10^{-6}$. The total scattering and absorption losses at both mirrors is $\delta_{0} \approx 44 \times 10^{-6}$. The cavity finesse is $\mathcal{F} \approx 15000$. The cavity is probed with a weak field incident on mirror $M_{1}$ with transmission through mirror $M_{2}$ recorded on a balanced heterodyne detector. From the size of the heterodyne photocurrent beat note between the probe and the detuned local oscillator (LO), the average intracavity probe field amplitude $\left|\left\langle a_{p}\right\rangle\right|$ can be inferred given the measured length and transmission of the cavity and the measured heterodyne detection efficiency. The experiment consists of recording values of $\left|\left\langle a_{p}\right\rangle\right|^{2}$ for a variety of parameters and scenarios. The squeezed light is incident on mirror $M_{2}$.

For the $6 S_{1 / 2}, F=4, m_{F}=4 \rightarrow 6 P_{3 / 2}, F^{\prime}=5, m_{F}^{\prime}=5$ transition in Cs, the atom-cavity rates are $\left(g_{0}, \kappa, \gamma_{\perp}\right) / 2 \pi=(20$, $80 \pm 5,2.5 / \Gamma) \mathrm{MHz}$, where $\Gamma=0.7$ accounts for a broadening due to the finite transit time of atoms across the cavity mode

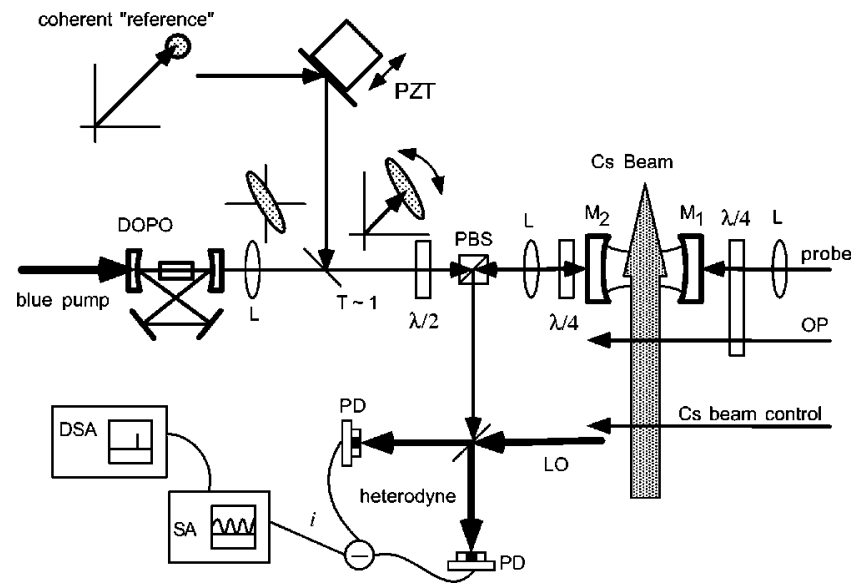

FIG. 4. Squeezing on atom-cavity experimental schematic: probe transmission spectrum measurements. The elements are as follows. OP, atomic beam optical pumping for preparation of twostate atoms; $\lambda / 4$, quarter-wave plate for preparation of $\sigma^{+}$-polarized light for excitation of the two-state atoms; $\lambda / 2$, halfwave plate for polarization preparation of the squeezed field; PBS, polarizing beam splitter for lossless splicing of the squeezed field into the $1 \mathrm{D}$ atom cavity; $L$, mode-matching lens; LO, balancedheterodyne local oscillator; PD, balanced-heterodyne photodiode; SA, spectrum analyzer set to measure the beat note between the LO and probe; DOPO, degenerate OPO for squeezing generation; the "Cs beam control" controls the number of atoms interacting with the cavity mode by adjusting the $F=4$ ground-state population. In addition, for excitation with displaced squeezed light (rather than squeezed vacuum) we add the DSA, the dynamic signal analyzer used to measure the power in the probe-modulation recorded by the $\mathrm{SA}$, and the PZT, the piezoelectric transducer used to adjust the relative phase between the reference field and the squeezed field.

[5]. [Note that $\Gamma$ affects the width of the absorption dip since from Eq. (1) $\gamma_{\perp}^{c}=\gamma_{\perp}+g_{0}^{2} / \kappa$.]

The probe beam is tunable in both power and frequency via the power and frequency of a rf sideband of a travelingwave modulator. (The optical power is always kept at a level such that the intracavity photon number due to the probe is less than the saturation photon number $m_{0}=4 \gamma_{\perp}^{2} / 3 g_{0}^{2}$, that is, $\left\langle a^{\dagger} a\right\rangle_{p}<m_{0} / 100, m_{0} \sim 10^{-2}$. This constitutes a weak probe that will not disturb the underlying structure of the system under investigation. Of course, the measured quantity is ultimately the result of the fact that the system is driven, so a complete theory that includes this fact must be developed.) The cavity length is locked such that a $\mathrm{TEM}_{00}$ mode is precisely resonant with the atomic transition $\left(\omega_{A}=\omega_{c}\right)$ with a strong locking beam that is switched off during data taking cycles. These aspects of the experiment have changed little from a previous experiment described in Ref. [5].

\section{Complications from the atomic beam}

Ideally, in the perfect version of our experiment, one envisions a single atom at the center of the cavity mode, optimally coupled to the cavity and interacting with the otherwise unaffected squeezed field that was coupled into the cavity. However, in practice the situation is far more complicated. The atoms propagate from a thermal effusive source forming a continuous stream of atoms that cross the Gaussian standing-wave cavity mode. At any instant in time, 
though the effective number of atoms may be approximately one $\left(N_{e} \approx 1\right)$, the cavity volume (the physical space between the two mirrors) is actually filled with far more than one atom. The effective number of atoms is a quantity measured by fitting the measured linear probe spectrum to an analytical expression, valid in the weak field $[5,98,99]$. This determines the collective coupling of a sample of atoms, which we call $C_{\text {meas }}, C_{\text {meas }}=N_{e} C_{1}$. Of course, we are interested not in the response of a collection of atoms to the squeezed-light excitation, but rather in the response of a single atom. That we actually do measure the response of a collection of atoms or, more accurately, that we are able only to measure the response of a single atom on those occasions when the random fluctuations of the atomic beam allow causes some difficulty in the interpretation of the results and in fact leads to a severe degradation of observable effects.

We call those atoms that are weakly coupled to the cavity field due to their positions on the wings of the Gaussian or near the nodes of the standing wave spectator atoms. These spectator atoms are each weakly coupled to the cavity, though collectively they can couple with strength comparable to the coupling of a single atom in an optimal part of the Gaussian standing wave. We define the saturation photon number associated with each of the spectator atoms as $m_{s_{i}}$ $=2 \gamma_{\|} \gamma_{\perp} / 3 g_{s_{i}}^{2}$, where $g_{s_{i}}$ is the coupling of spectator atom $i$, and the collective cooperativity of all the spectator atoms as $C_{s}=\left(1 / 2 \kappa \gamma_{\perp}\right) \Sigma_{i} g_{s_{i}}^{2}$. The concept of a collection of atoms weakly coupled to the cavity and an atom strongly coupled implies a division in coupling-space. We can define two effective mode volumes (integrals over the mode functions for certain regions of coupling space): $V_{\text {in }}$, for atoms that satisfy a minimum coupling requirement, and $V_{\text {out }}$, for atoms that do not satisfy this minimum coupling requirement. For a uniform density atomic sample, the ratio of the "in" and "out" mode volumes determines the ratio of collective couplings of the "good" atom(s) and the spectator atoms, respectively. For a boundary defined by $g_{s_{i}}^{2} / g_{0}^{2} \sim(0.56)^{2}$, this ratio is $V_{\text {in }} / V_{\text {out }} \sim 1$. Hence we say that the spectator atoms give rise to an effective spectator coupling of $C_{s} \approx C_{\text {meas }} / 2$. Note that this definition also agrees with a threshold used in several of our previous experiments $[98,99]$.

Because each spectator atom is weakly coupled (it turns out that the average coupling of each spectator atom is $\bar{g}_{s}$ $\sim 10^{-2}$ ), its saturation photon number is very large compared to that of the near-optimal single atom $\left(m_{s_{i}} \gg m_{0}\right)$ and it is not expected to contribute to the "interesting" effects of the system, such as nonlinear response and effects due to the squeezed field. The spectator atoms can, however, act as a loss mechanism, absorbing the squeezed field without contributing to observable effects due to the statistics of the squeezed field. In this approximation, the spectator atoms taken collectively are very much akin to a simple intracavity loss, such as that contributed from the scattering and/or absorption losses in the mirror coatings described by $\delta_{0}$. We can call such a loss term $\delta_{A}^{\mathrm{spec}}$. We will return to this in Sec. II C. Thus, for an experimentally measured value of $C_{\text {meas }}=N_{e} C_{1} \approx 1$, actually about half of the coupling strength is due to spectator atoms that behave as a linear medium unaffected by the squeezed light, but absorbing it nonethe- less. The other half of the coupling is the average coupling (over both space and time) due to a single atom strongly coupled (in the sense defined above) to the field mode, whose behavior in the presence of squeezed light we wish to determine. We will show in Sec. IV that the above argument is fairly well supported by the results of our experiment when compared with the simplified theory (not including atomic beam effects) presented in Sec. III. Needless to say, this nonideal circumstance is an added complication that we are actively working to eliminate by trapping and localizing single atoms on the intense parts of the intracavity field $[100,101]$.

\section{B. Laboratory 2: Squeezing}

As described in Refs. [95-97], for the tunable squeezed light at the 852-nm transition wavelength of atomic Cesium which we employ, a Ti:sapphire laser locked to a Cs cell at $852 \mathrm{~nm}$ pumps an external doubling cavity to generate frequency-doubled blue light at $426 \mathrm{~nm}$ [97]. This doubled light pumps an OPO operated below threshold, which downconverts one high-energy photon into two correlated lowenergy photons generating a close-to-minimum-uncertainty squeezed-vacuum state. The OPO cavity bandwidth (FWHM) is $\kappa_{s}=2.4 \gamma_{\|}=2 \pi \times 12 \mathrm{MHz}$, which is less than $2 \gamma_{\perp}\left(1+2 C_{1}\right)$, the full width of the cavity-enhanced 1D atom. Therefore, the usual broadband treatment of the squeezed light-atom interaction is inadequate for the purposes of describing the dynamics of our experimental realization and a more complicated theoretical approach must be taken to account for the finite bandwidth of the squeezed light.

The OPO is capable of producing squeezed light with detected noise level reduced by $6 \mathrm{~dB}$ below the shot-noise level, near the OPO cavity peak [95]. The gains that can be reached in practice range over $1<G_{+}<20$. The squeezed field typically carries a few picowatts of optical power. At the output of the OPO, we mix on a 99-1 beam splitter the squeezed vacuum with a phase-coherent reference oscillator with controlled relative phase to the squeezing, resulting in a combined electromagnetic field that is equivalent to a displaced squeezed state. The displacing reference field can have virtually arbitrary power $P_{\text {ref }}$, but is typically restricted to $P_{\text {ref }}<200 \mathrm{pW}$ since at this point the $1 \mathrm{D}$ atom is saturated.

\section{Getting the squeezing into the cavity and onto the atom}

\section{Squeezing buildup in the presence of an atom}

To our knowledge, a rather important practical issue has been left mostly unconsidered in the literature. This is the issue of getting the squeezed light from an external source into the atom-cavity system. In much of the work done on the subject of squeezed light interaction with atoms in cavities, the squeezing is inserted in a rather idealized way directly into the cavity. In this picture, as in Ref. [58], the effects of the squeezed light (whatever they happen to be) are always better the larger the single-atom cooperativity parameter $C_{1}$ is made. However, if we consider that the atom (for this argument a single, fixed, optimally coupled atom) can be treated as a lossy intracavity medium with associated loss parameter in the weak-field limit given by [102] 

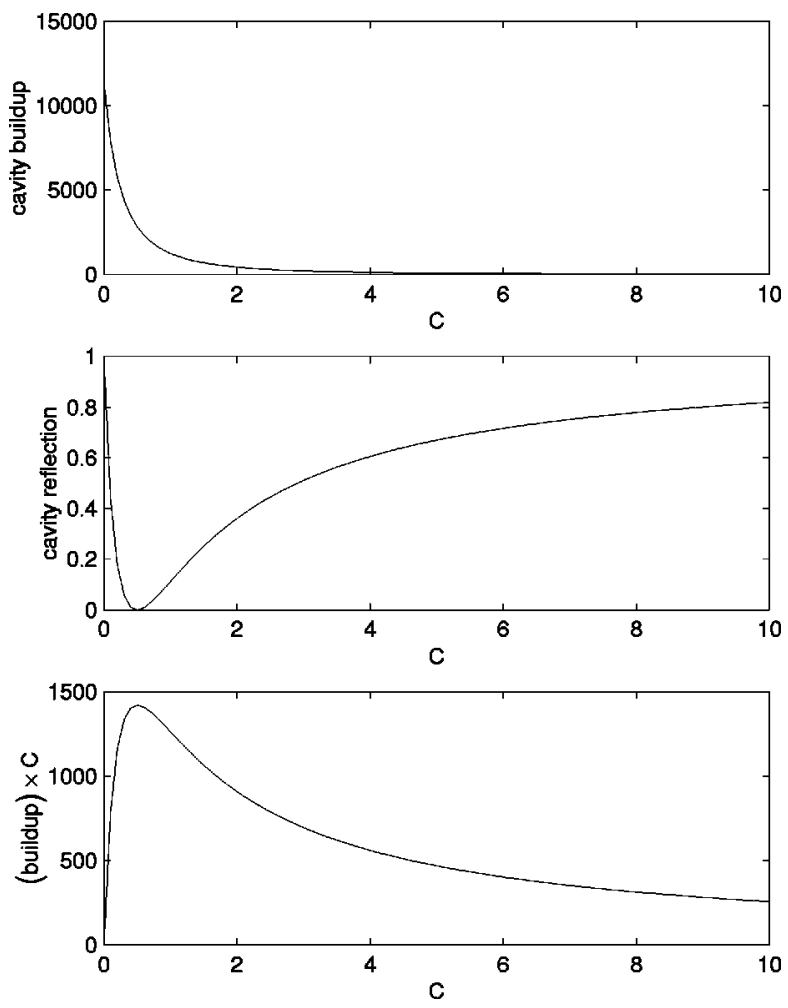

FIG. 5. Cavity buildup $\mathcal{B}$, reflection $\mathcal{R}$, and buildupcooperativity product $\mathcal{B} C$ vs $C$ for a weak coherent state resonant with the common atom-cavity frequency. The buildup is given by Eq. (28) with Eq. (27) and the reflection is given by Eq. (29) with Eq. (27) with $\delta_{2}=350 \mathrm{ppm}, \delta_{1}=1 \mathrm{ppm}$, and $\delta_{0}=0$. The product buildup $\times$ cooperativity $(\mathcal{B C})$ is discussed in the text.

$$
\delta_{A}=2\left(\delta_{1}+\delta_{2}+\delta_{0}\right) C_{1},
$$

then it is not at all clear that large $C_{1}$ is the optimal approach. For large $C_{1}$, the associated intracavity losses $\delta_{A}$ are also large, so the squeezed field does not actually build up in the cavity. To emphasize this point, we have plotted in Fig. 5 the cavity buildup $\mathcal{B}$ and the cavity reflectivity $\mathcal{R}$ as $C$ is increased [just replace $C_{1}$ by $C$ in Eq. (27)]. As a reminder, for a resonant (coherent-state) field incident on mirror $M_{2}$ (as is the squeezing),

$$
\begin{aligned}
& \mathcal{B}=\frac{4 \delta_{2}}{\left(\delta_{1}+\delta_{2}+\delta_{0}+\delta_{A}\right)^{2}}, \\
& \mathcal{R}=\frac{\left(\delta_{1}+\delta_{0}+\delta_{A}-\delta_{2}\right)^{2}}{\left(\delta_{1}+\delta_{2}+\delta_{0}+\delta_{A}\right)^{2}} .
\end{aligned}
$$

For the plots of Fig. 5 the cavity is one sided, with the input mirror $\delta_{2}=350 \mathrm{ppm}$, the essentially perfect back mirror $\delta_{1}$ $=1 \mathrm{ppm}$, and $\delta_{0}=0$, for simplicity. From Fig. 5 we clearly see the expected behavior in that the buildup decreases as $C$ is increased. This does not really help us determine an optimal value of $C$, however, as the buildup is largest when there is no atom in the cavity. The cavity reflection does, however, imply an optimal value of $C=1 / 2$. At this point, there is no squeezed light reflected from the cavity, so in some sense, the most squeezing possible is building up in the cavity (in the presence of an atom) and interacting with the atom. This idea can be further quantified in the third and final graph of Fig. 5. We have seen that the qualitative effect of the squeezing is to modify the cavity-enhanced decay rate of the atom: $\beta_{ \pm}^{c}=\gamma_{\perp}\left[1+2 C_{1} \Delta X_{ \pm}\right]$. The squeezing variance $\Delta X_{ \pm}$is the one that actually builds up in the cavity. In our experiment, we have an external squeezing with variance $\Delta X_{ \pm}^{\text {ext }}$, which then builds up in the cavity in the presence of the atom to a value $\Delta X_{ \pm}=\left(\mathcal{B} / \mathcal{B}_{0}\right) \Delta X_{ \pm}^{\text {ext }}$ (in a qualitative sense), where $\mathcal{B}$ is the cavity buildup with losses (the atom) and $\mathcal{B}_{0}$ is the peak cavity buildup in the absence of internal losses (no atom). The product $\mathcal{B C}$ thus determines the effect on the 1D atom from an external squeezed field. This is plotted in the bottom graph of Fig. 5; it is also seen to be optimal for $C=1 / 2$. The value of $C=1 / 2$ is a generic feature of the one-sided cavity driven through the high transmission mirror, as can be seen from Eq. (27) in Eq. (29). This is, of course, the case of the impedance-matched resonator. That our $1 \mathrm{D}$ atom has $C_{1} \approx 1$ is quite fortuitous from this perspective. This treatment points out the fact that there is little to be gained in terms of the potential effects of the squeezing by increasing $C_{1}$, a point that is completely at odds with a theory that does not consider an external source of squeezing. This is further supported by the theory of Sec. III.

\section{Intracavity losses due to the spectator atoms}

As an extension of the above line of reasoning, we can immediately get a qualitative (and quantitative) understanding of the detrimental effects of the spectator atoms. These atoms contribute an additional loss mechanism, without being sensitive to the photon statistics of the squeezed field. They will allow the intracavity squeezed field to build up only according to Eq. (28) with a $\delta_{A}^{\text {spec }}$ that arises from the cooperativity parameter of the spectator atoms $C_{s}$, as discussed above. This loss term is given as the ratio in buildup for a near-optimally coupled atom in the presence of spectator atoms to a near-optimally coupled (and fictitious) atom with no spectator atoms present. We call the coupling of the near-optimally coupled atom $C_{n}$. The buildup of squeezing with this atom alone goes as $\left(1+2 C_{n}\right)^{-2}$, while the buildup of the squeezing with this atom and the spectator atoms scales as $\left[1+2\left(C_{n}+C_{s}\right)\right]^{-2}$. Thus an effective loss due to the spectators is

$$
\eta_{\mathrm{spec}}=\left(\frac{1+2 C_{n}}{1+2\left(C_{n}+C_{s}\right)}\right)^{2}
$$

Now, by caveat, $0.3<C_{n}<1$ [over our mode volume $C_{n}$ $\left.>(0.56)^{2} C_{1}=0.3 C_{1}\right], C_{s}=C_{\text {meas }} / 2$, and in our experiment $1.24>C_{\text {meas }}>0.55$. Over this range of numbers $\eta_{\text {spec }}$ varies by $0.41 \pm 0.09<\eta_{\text {spec }}<0.63 \pm 0.08$, which will be used later. This loss due to the spectator atoms is quite significant and likely to be the single greatest problem with the experiment. In this light, we have attempted to run the experiment with $\bar{N} \approx 0.5$, where the contribution of the spectator atoms is smaller. We have not found, however, an optimal balance between the competing effect of a reduced loss contribution from spectator atoms and the reduced signal-to-noise ratio (SNR) accompanying the smaller values of $N_{e} C_{1}$. Indeed, there may not exist any easy way around this, beyond realizing an optimal system in which a single, stationary, and localized atom is employed. 


\section{Measurements}

We have made two types of measurements of the 1D atom in the presence of squeezed fields. The first probes the transmission spectrum of the 1D atom, essentially spectroscopy of the $1 \mathrm{D}$ atom in the presence of a squeezed reservoir for the atom. In order to make direct experimental comparisons with other types of light, we have performed spectroscopy of the 1D atom with an applied thermal reservoir with identical properties (spectral content, i.e., power, bandwidth, and line shape) as the squeezed one. In the second type of measurement we fix the frequency of the probe field and monitor the modulation in transmission as we rotate the phase of the squeezed field with respect to a saturating coherent "reference" field (displaced squeezing). We have measured the size of the transmission modulation as a function of a wide variety of system parameters such as the strength of the saturating reference field, detuning of the probe, and degree of squeezing. In this section we will limit the discussion to the measurements, leaving the theoretical discussion for the next. A discussion of the comparison of theory and data will be deferred until Sec. IV.

\section{Measurements of probe transmission spectra with squeezed and thermal reservoirs}

The experimental arrangement for the probe transmission spectrum measurements is shown schematically in Fig. 4. A weak probe is scanned in frequency across the atom-cavity resonance $\left(\omega_{A}=\omega_{c}\right)$. The transmission is measured both with and without the squeezed vacuum input. The squeezed vacuum (no coherent reference field present) enters the cavity through the output coupler $M_{2}\left(\delta_{2}=322 \mathrm{ppm}\right)$ of the cavity. The polarizations are arranged so that the squeezed field has the same helicity $\left(\sigma^{+}\right.$polarization) as the probe field. Because of this, the squeezed light bounces off the cavity and is reflected along the same path as the probe transmission and will be detected at the heterodyne detector. The squeezing beats against the LO and has power spread over some bandwidth given by $\kappa_{s}$, giving rise to what amounts to a measurement of the spectrum of output power of the OPO. This "measurement" was used to ensure that there was actually light from the OPO and that the detuning of the squeezed field was near zero. This detuning tended to drift during the experiment, so it was checked frequently, resulting in these slow excursions from the atom-cavity resonance $\left(\omega_{A}=\omega_{c}\right)$ being kept to $\pm 2 \mathrm{MHz}$. The squeezing was measured by homodyne in both laboratories 1 and 2 to confirm the existence of squeezing as close to the $1 \mathrm{D}$ atom as possible. We will quote the OPO gain $G_{+}$as a measure of the squeezing since the homodyne was not used on a daily basis.

The heterodyne detector is sensitive to all the incident fields, so a procedure of subtracting various quantities was employed to extract the normalized atom-cavity transmission $T_{n}=T_{\text {with atoms }} / T_{\text {no atoms }}$ in the presence of squeezing $\left(T_{s}\right)$ and without squeezing $\left(T_{v}\right)$. Note that the absolute size of the effect due to squeezed excitation is larger in all cases than these corrections.

Additionally, we can define the quantity $\Delta_{s}$,

$$
\Delta_{s} \equiv T_{s}-T_{v},
$$

as the difference between the spectra with and without squeezing. In the absence of generic theoretical criteria that provide unequivocal tests of the nonclassical effect of squeezed light on an atom in a cavity (especially for our particular realization of that system, including the atomic beam and other complications) it is essential (and preferable) to find measurements that differentiate light of various quantum (or classical) character. We have done this by exciting the atom cavity with both squeezed vacuum and thermal light from a single mode of the nondegenerate OPO (NOPO). If the degenerate OPO is locked off of the Cs transition by one longitudinal mode spacing (650 MHz away) such that the next longitudinal mode is resonant with the Cs transition, then quantum correlations of the down-converted light will be between one photon that is resonant with the Cs transition and one photon that is $1300 \mathrm{MHz}$ detuned from the Cs transition. Thus, as far as the atom is concerned, the squeezing will be lost and the 1D atom will be illuminated with a purely thermal mode. The key to this technique is that the thermal light from a single mode of the NOPO is identical in all respects to the squeezed light from the degenerate OPO, except for the classical statistics of the former and the quantum statistics of the latter. For the same phase-sensitive OPO gain, the bandwidth of both sources is the same and the number of photons in each source is the same. For purposes of comparison, we define the quantity analogous to $\Delta_{s}$ of Eq. (31) for thermal light and call it $\Delta_{t}$. The shape of $\Delta_{t}$ has distinguishing features that make it quite distinct from $\Delta_{s}$ in the ideal case.

The effect on the probe transmission spectrum of the squeezed (or thermal) light proved to be rather small, making the experiment a difficult one, ultimately incapable of distinguishing the probe spectrum in the presence of a squeezed field from that of excitation in the presence of a thermal field. Figure 6(a) is $\Delta_{s}$ for squeezed input and Fig. 6(b) is the corresponding $\Delta_{t}$ for an "identical" thermal input, for data taken over a similar range of power $P$, where $P$ is a relative measure of the power in the squeezed (or thermal) field (determined by measuring the height of the noise spectrum due to the squeezed field on the heterodyne detector), with $P$ $\approx 1$ roughly equivalent to a gain $G_{+} \approx 5$ (little effort was made to establish the exact conversion since only relative comparisons between thermal and squeezing are relevant).

All the difference spectra (Fig. 6) are averaged without regard for experimental parameters (the OPO gain being the principal one) and are shown in Fig. 7. We have argued [103] that the shape of these curves is different, especially in the wings, where the thermal difference spectrum dips below that of the squeezing, as would be expected (see Fig. 16), but the results are certainly not stunning.

It is clear given the size of the modifications in the probe spectra that we observe that there is little hope of measuring reduced linewidths due to the squeezed reservoir (or enhanced linewidths due to the thermal reservoir). But why is this? For our parameters and perfect squeezing, the reduction in the linewidth should be a factor of 3, according to Eq. (24). Even accounting for our nonperfect squeezing, 50\% squeezing should give a $30 \%$ reduction and bandwidth effects should have only minimal impact. The results of the measurements would seem to imply that there is some loss involved in coupling the squeezed light to the atom, above and beyond easily measured losses such as transport loss and 

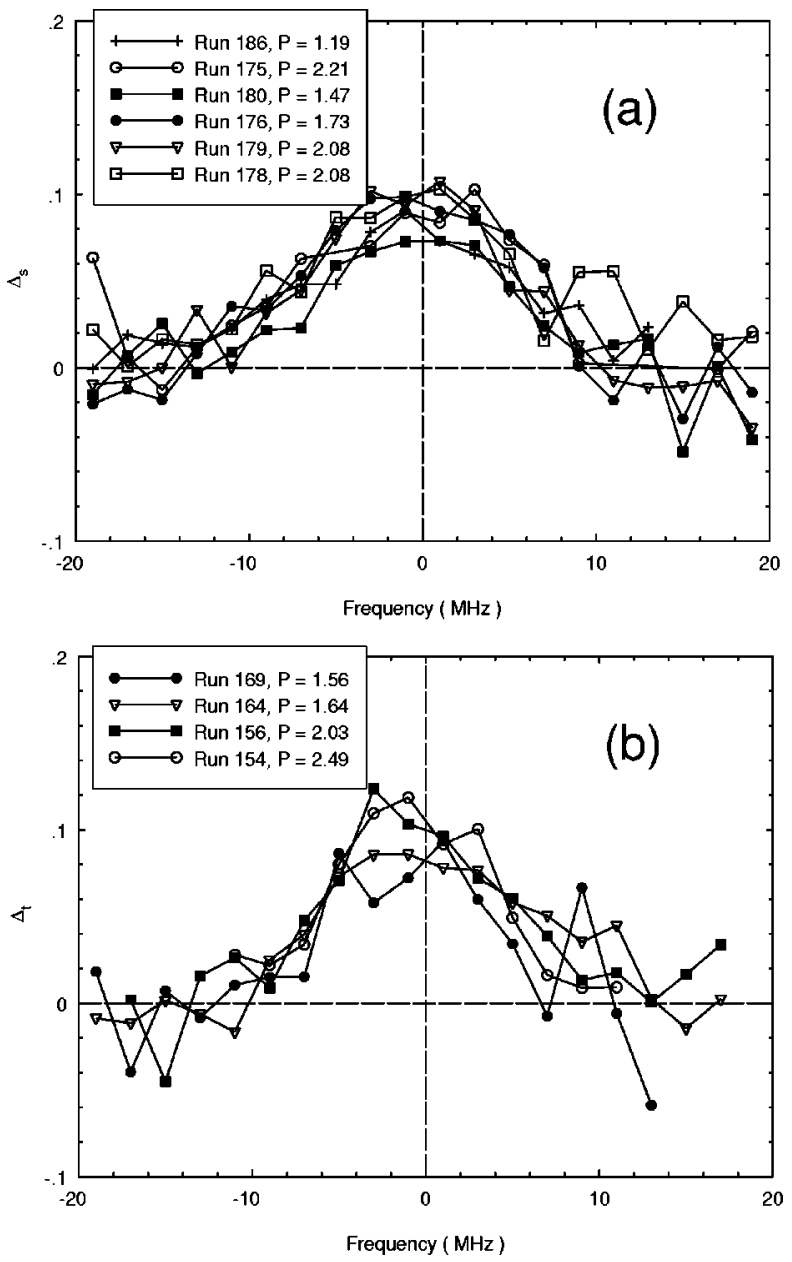

FIG. 6. (a) Difference spectrum $\Delta_{s}$ with squeezed light excitation. (b) Difference spectrum $\Delta_{t}$ with thermal light excitation. The coincident atom-cavity resonance is at $0 \mathrm{MHz}$.

cavity mode-matching loss, etc. Within the context of the discussion of Sec. II C, particularly the effects of the spectator atoms, this additional loss mechanism now seems clear, though it was by no means so in the beginning stages of the experiment. The discussion of these details will be resumed in Sec. IV.

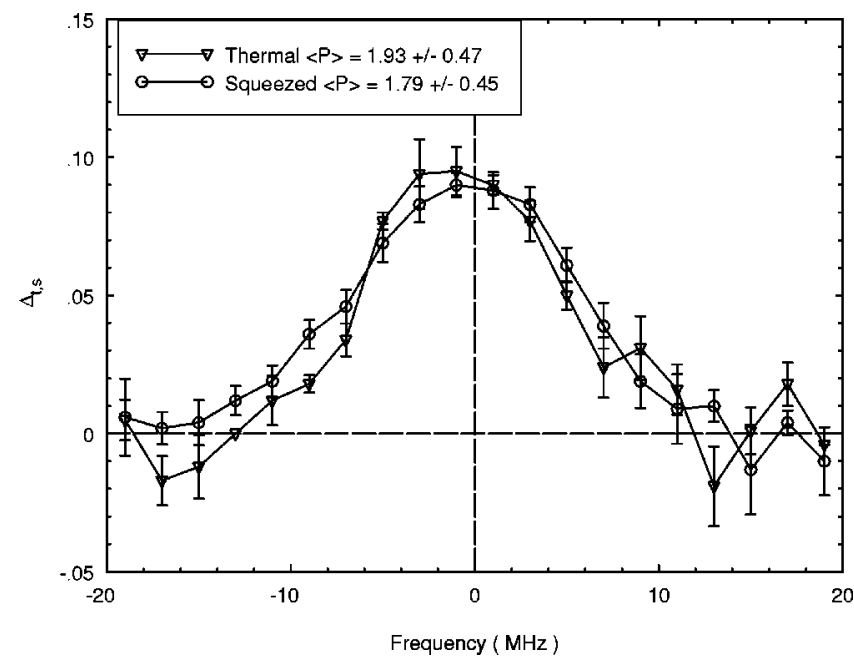

FIG. 7. Averaged difference spectra, thermal and squeezed. The coincident atom-cavity resonance is at $0 \mathrm{MHz}$.
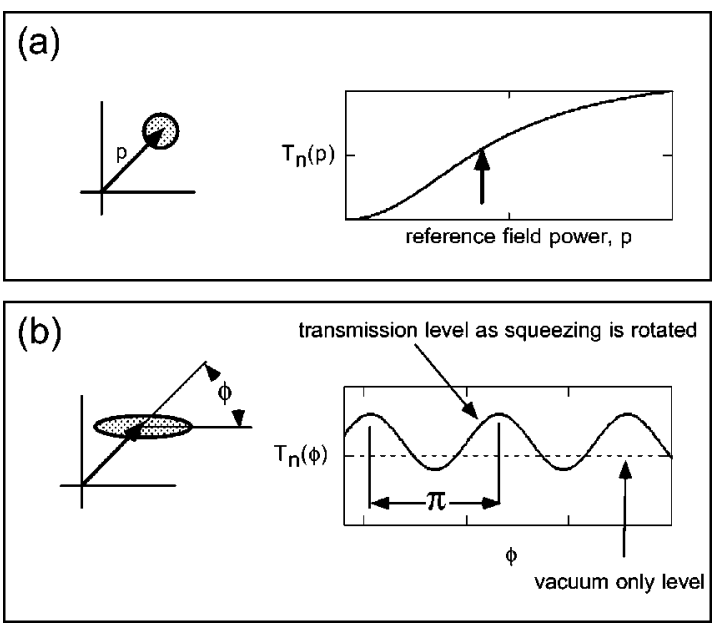

FIG. 8. Idea of the phase-sensitive transmission measurement.

\section{Measurements of phase-sensitive probe transmission with squeezing}

The measurements of the transmitted probe spectrum of the atom cavity in squeezed vacuum were difficult and inconclusive, as seen above. In pursuing a second measurement strategy, which attacks the problem from a completely different angle, it was hoped that an effect of the squeezing would be more pronounced or more robust against complications of the experiment.

In this scheme, there are again two fields incident on the atom cavity. In this case, one is a displaced squeezed field. For concreteness, we will call the resonant coherent displacing field the reference field. The other incident field is the fixed frequency, fixed power (weak) probe. We measure the response of the weak probe transmission to the modulation of the relative phase of the squeezed vacuum output of the $\mathrm{OPO}$ with the reference (displacing) coherent field for various parameters of the system. There are three parameters that we can change: the degree of squeezing (as quantified by the OPO gain $\mathrm{G}_{+}$), the amplitude $\Omega$ of the reference field, and the detuning of the probe field. An additional parameter that we can change is the effective number of atoms present in the cavity, although for this control parameter we only have a limited number of data points.

Now consider the schematic of Fig. 8. We probe the 1D atom with the weak probe. First we apply the resonant coherent reference field to saturate the atoms to some desired level (a). [Figure 8(a) is a qualitative plot of the normalized transmission as a function of reference field power. It displays the saturation behavior of the 1D atom. For measurements and a further description of this property, see Refs. $[90,102,104]$.$] In the presence of the reference field (and$ normal vacuum), the transmitted probe field will be at some dc level indicated by the dashed line in Fig. 8(b). Next we apply the squeezed field and monitor the probe transmission as a function of the phase of the squeezed field with respect to the reference field. The transmitted probe field will oscillate at twice the frequency at which the squeezing is rotating around the coherent reference field indicated by the hypothetical solid curve in Fig. 8(b). Let us emphasize that for a weak probe, which we are considering here, the modulation of the probe will be independent of its own strength, but not independent of the strong (saturating) reference field 
strength. The reason for the modulation of the probe transmission can be understood as the direct response to a modulated saturation level that depends on the relative phase of the squeezed vacuum with respect to the reference field, through an alteration in $\gamma_{x, y, z}$ of Eq. (21), for example. In particular, for the case when the phase of the squeezed vacuum is such that the displaced squeezed state has excess noise along the reference field amplitude, one expects a higher degree of atomic saturation. In contrast, for the choice of phase for which the squeezed noise is suppressed along the reference field, the saturation decreases. Here we note that the above argument for modulation of the transmission holds true not only for the case where we displace the quantum squeezed vacuum but also if we have displaced a classical squeezed state (which also has asymmetric quadratures) or even in the simplest possible case if we mix the reference field with another phase coherent laser field and then vary their relative phase. Hence, as discussed earlier in the Introduction and Sec. I C, simply the fact that the transmission of the probe beam is modulated is by no means a proof of a nonclassical effect being observed.

The level of the transmitted probe field with squeezing will be different from the level without the squeezed field, which we will suggestively call the "vacuum-only" level (it is the transmission of the probe with only vacuum fluctuations on the coherent reference field). Could it be the case that a dip of the transmission below this vacuum-only level is one of the elusive indicators of the quantum nature of the squeezed field? In other words, is this level analogous to the vacuum level defined by the shot noise of a local oscillator on a photodiode? The answer is not completely straightforward. Apparently, in some cases it is; in some cases, it is not. In Sec. III we will explore the relevant parameter space to find those locations in which the vacuum-only level is an indicator akin to the shot noise of a homodyne squeezing measurement. We stress at this point that the measure of a nonclassical effect that we are considering is not identical to a measurement in which the noise spectrum of a squeezed field is directly compared to the shot-noise level of a LO. Here we are considering the probe transmission as a measure of the potentially nonclassical interaction of the squeezed vacuum and the $1 \mathrm{D}$ atom.

The apparatus for the phase-sensitive measurements is shown in Fig. 4. The phase of the squeezing relative to the reference field is controlled by the piezoelectric transducer (PZT). The reference field is mixed with the squeezing on a beam splitter, which induces negligible loss to the squeezing.

Based on the expected size of the modulation signal (up to 25\% modulation in Fig. 15, e.g.), the measurement should have been straightforward; it turned out not to be. The measurement procedure consisted of the following. As the phase of the squeezing is rotated with respect to the coherent reference field with the PZT of Fig. 4, a modulation in the probe transmission is monitored as changes in the probe-LO beat-note size on the spectrum analyzer (SA). Unfortunately, this signal was too small to be resolved in the time domain, but was well resolved in the frequency domain by sending the rf-demodulated beat-note signal from the SA video out to a Hewlett Packard model HP3562A dynamic signal analyzer (DSA), which was used essentially as a low-frequency spectrum analyzer. The DSA registers a peak in the power spec-

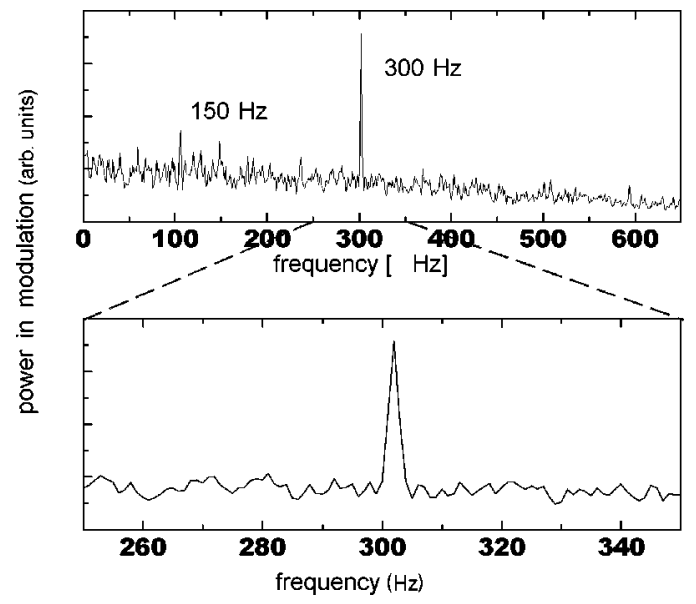

FIG. 9. Signal from the DSA, after rf demodulation from the SA. Peaks such as this for a variety of parameters are used to infer $\alpha_{p p}$.

trum corresponding to twice the frequency $(2 f)$ at which the squeezing ellipse is rotated $(f)$. There is no peak when the atoms are not present, confirming that the modulation is not merely a result of the squeezing ellipse detected on the heterodyne (there is no simple way for this to be the case anyway). That there is also no peak at $f$ indicates that the transmission modulation is not due to, e.g., a misalignment of the reference beam as the PZT is scanned (the scan distance is only on the order of the optical wavelength). The nominal frequency of squeezing-ellipse rotation is determined by making an interferometer with the coherent reference and an auxiliary coherent beam on the same path as the squeezing. A typical DSA trace is shown in Fig. 9. The peak at $300 \mathrm{~Hz}$ shows that the probe transmission does indeed have frequency content at twice the frequency of rotation of the squeezing ellipse, which is independently determined to be $150 \mathrm{~Hz}$.

Note that while the frequency-domain SNR is respectable, we were never able to resolve directly the modulation in the time domain. Therefore, due mainly to technical limitations, we are unable to measure the probe modulation with respect to the vacuum-only level. We can, however, measure the peak-peak magnitude of the modulation. If we define $A_{P}(\nu)$ as the transmitted amplitude of our weak probe beam, then a measure of the magnitude of the peak-peak probe modulation is conveniently given by the "amplitude fractional modulation," defined as

$$
\alpha_{p p}=\frac{\left|A_{P}^{+}(\nu)-A_{P}^{-}(\nu)\right|}{\left|A_{P}^{\text {no sqz }}(\nu)\right|},
$$

which is normalized to the vacuum-only level $A_{P}^{\text {no sqz }}(\nu)$. The plus and minus refer to orthogonal phases of the squeezing (the case where the displaced squeezed state has noise excess and noise suppression, respectively), which we assume to be correlated with the maxima and minima of the probe modulation. This assumption is justified by the theory and is confirmed by the appearance of the peak in the modulated-probe power spectrum at $2 f$. For resonant probe measurements $\nu=0$. 


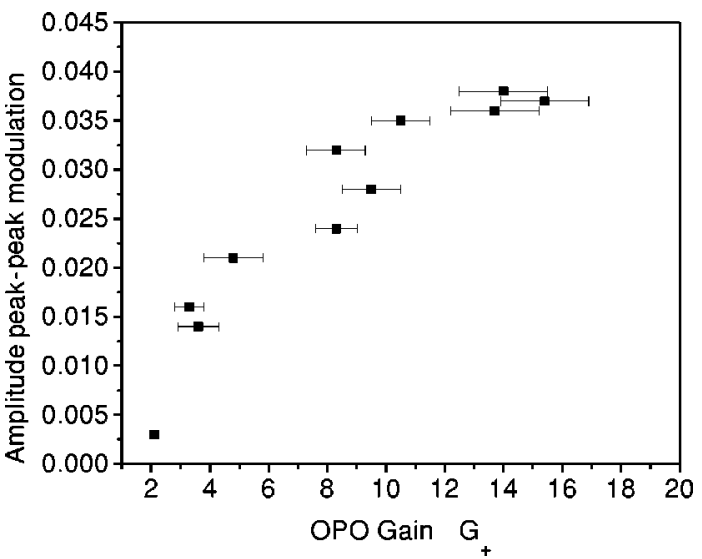

FIG. 10. Amplitude fractional modulation $\alpha_{p p}$ vs OPO gain $G_{+}$. The reference power is $P_{\text {ref }}=50 \mathrm{pW}$ and $\bar{N}=0.7$ atoms.

Unfortunately, in this experiment we had no direct means to compare the quantum system with a classical system as we did in the spectra measurements. The logical choice would have been classical squeezing as discussed in Sec. III, but a source of this type of light was not realized. Nonetheless, we were able to map out the behavior of the system under variation of many parameters and the results agree quite well with the functional form of predictions. The absolute magnitude of the effects was, however, significantly below theoretical predictions for an ideal system of a single atom in a cavity; however, the predictions are actually quite good if all loss mechanisms are accounted for in the treatment. This will be discussed in Sec. IV.

We now turn to the measurements and the data. The displaced squeezed field is nominally at the frequency of the common atom-cavity resonance. We attempted to avoid contributions to the signals from the spectator atoms by going to ever smaller mean atom numbers. It was typical for us to work in the regime of $\bar{N} \approx 0.5$ atoms. At this operating point the influence of spectator atoms is smaller, but of course hand in hand with this is that the empty cavity is a dominant contribution and the overall size of the effect is smaller.

Figure 10 shows the amplitude fractional modulation $\alpha_{p p}$ of Eq. (32) as a function of the OPO gain $G_{+}$for a fixed coherent reference power of $25 \mathrm{pW}$. The coherent reference power is measured via its beat note with the local oscillator on the heterodyne detector, which is calibrated to account for efficiency of overlap of these beams (which are generated from independent lasers). We expect that the probe transmission modulation will be larger as the degree of squeezing is made larger, as this causes the largest asymmetry between the quadrature amplitudes of the squeezing. On the other hand, the fractional modulation eventually saturates, in keeping with the idea that the effect of the squeezing is not limitless, as seen in Figs. 14 and 15. Thus the trend of the data is certainly reasonable and in keeping with expectations, but the absolute magnitude is small (fractional modulation of only a few percent).

Figure 11 (top) shows the amplitude fractional modulation $\alpha_{p p}$ as the reference power is increased for fixed OPO gains of $G=5$ and 10 (for slightly different numbers of atoms). Clearly, if the reference field is acting as sort of a local oscillator for the squeezing (via its saturation of the probe
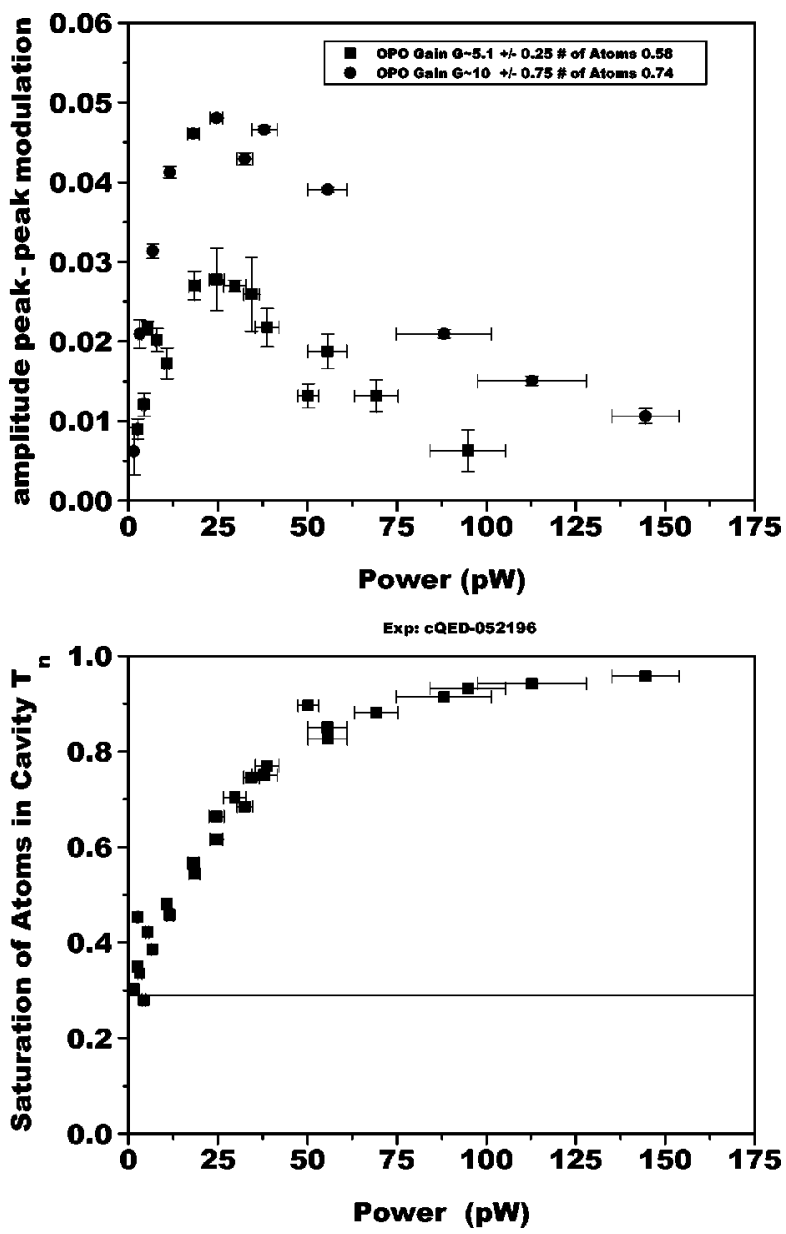

FIG. 11. Amplitude fractional modulation $\alpha_{p p}$ vs coherent reference power (top). Saturation of the normalized probe transmission $T_{n}$ vs coherent reference power, no squeezing, for the same parameters as above (bottom). $\bar{N}=0.6$ atoms for both figures.

transmission), its level must have an effect on the probe transmission that is larger than the modulation itself. Indeed, the modulation does increase as the reference field increases. The modulation amplitude reaches a peak somewhere near the point at which the probe transmission is halfway between its value for an empty cavity and its value for atoms in the cavity with no applied reference field. Again, this is probably not surprising, as the saturation slope is steep at this point and levels off in either direction. However, again, in keeping with the idea of the reference field as a local oscillator, the "'detector' (in this case the probe transmission of the atom cavity) eventually saturates to too large a degree and the modulation is no longer present. The data certainly support this idea. The saturation curve for the probe transmission over the same range of coherent reference power (in the same units) with no squeezed field is shown in Fig. 11 (bottom) for comparison. (Note that the probe is of fixed weakfield power in this trace; it is the reference field power that is being varied.) This is simply the saturation of the "detector'" by the local oscillator. Thus the data show the intuitive trends, but are much smaller in absolute size than expected.

Figure 12 shows the amplitude fractional modulation $\alpha_{p p}$ as the probe detuning is varied for fixed reference power and OPO gain. The squeezing frequency is fixed on resonance. The effect of the squeezing is most noticeable on resonance, 


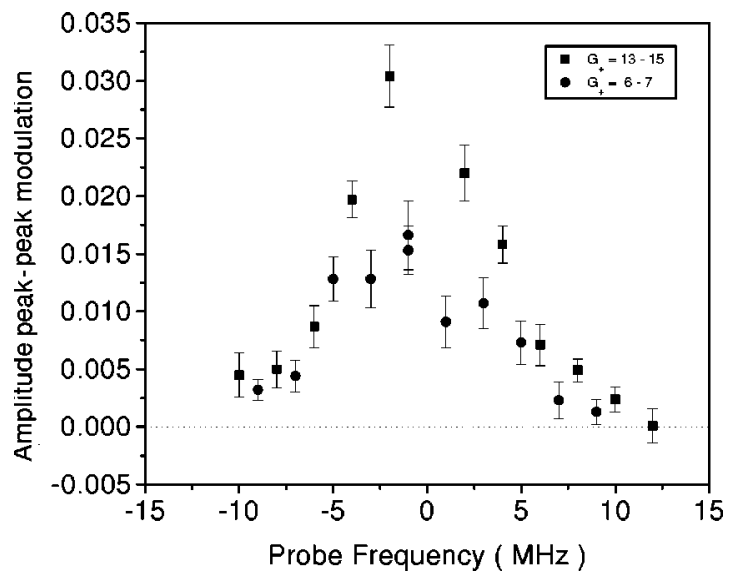

FIG. 12. Amplitude fractional modulation vs probe frequency. Reference power $P_{\text {ref }}=40 \mathrm{pW}\left(G_{+}=6.5\right.$ data $)$ and $P_{\text {ref }}=60 \mathrm{pW}$ $\left(G_{+}=14\right.$ data); the common atom-cavity center is at $0 \mathrm{MHz}$ and $\bar{N}=1.2$ atoms.

not surprisingly, and falls off away from the center frequency of both the squeezing and its "detector."

To complete the discussion, we also show the fractional amplitude modulation $\alpha_{p p}$ as the number of atoms is varied in Fig. 13. These data are taken over a collection of other parameters, hence the wide variation in $\alpha_{p p}$ at each atom number. We were unable to force the oven to produce any more than $\bar{N} \sim 2$ atoms, unfortunately, as it would have been interesting to mark the point at which the probe modulation disappears (assuming that it does in the many-atom case).

To conclude this section, we have measured the probe transmission modulation for a squeezed field rotating in phase with respect to a coherent reference field, whose action on the atom cavity is qualitatively similar to the mixing of a squeezed field with a local oscillator. The trends in the data are very much as one would expect, but the overall size of the effects is much smaller than anticipated. We defer a further discussion for Sec. IV, when the theory developed in Sec. III will be compared directly with the data.

\section{THEORY}

The goal of this section is not only to develop a (singleatom) theory that can account for the basic features of the experiment in a quantitative way, but also to develop a com-

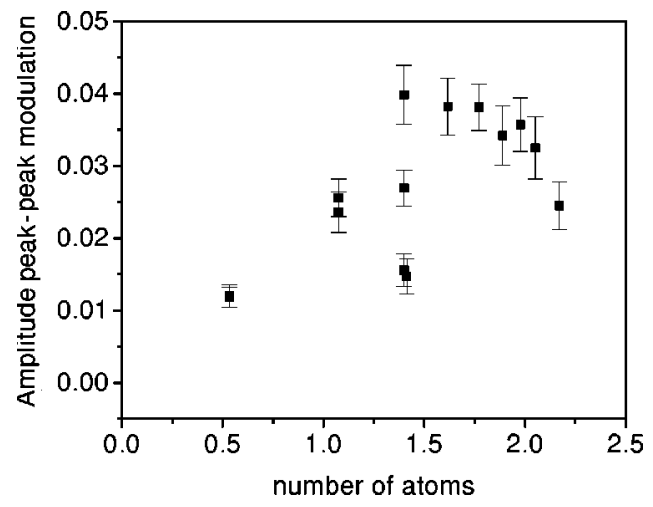

FIG. 13. Amplitude fractional modulation vs number of intracavity atoms. $0<G_{+}<20$ and $0<P_{\text {ref }}<150 \mathrm{pW}$. plete program of measurements that could in principle be made to determine whether our specific system behaves in a manifestly nonclassical way. In this light, we restrict our attention to properties that can be measured in a relatively straightforward manner, such as the probe transmission, while avoiding more complicated measurements such as two-photon correlation or two-time correlation measurements, which would also be viable approaches. The theory was largely developed while we still believed that we could measure the position of the modulated probe field with respect to the vacuum-only level, so a large portion of the treatment focuses on this issue. This measurement by itself can distinguish quantum effects from other types of effects under certain circumstances.

If we denote by $c$ and $c^{\dagger}$ the annihilation and creation operators for the cavity mode interacting with the atoms, then it can be shown that the transmitted amplitude of a weak probe incident on the cavity is proportional to the quantity

$$
A_{p}(\nu)=\int_{0}^{\infty} d \tau e^{i \nu \tau}\left\langle\left[c(\tau), c^{\dagger}\right]\right\rangle
$$

where $\nu$ is the frequency of the probe and angular brackets denote a stationary average. In the bad-cavity limit $(\kappa \gg g$ $\gg \gamma$ ), we can adiabatically eliminate the cavity mode; the correlation function appearing in the expression for $A_{\mathrm{p}}(\nu)$ can be approximated in this limit by

$$
\begin{aligned}
\left\langle\left[c(\tau), c^{\dagger}\right]\right\rangle & \simeq \exp (-\kappa \tau / 2)-\frac{4 g^{2}}{\kappa^{2}}\left\langle\left[\sigma_{-}(\tau), \sigma_{+}\right]\right\rangle \\
& \equiv \exp (-\kappa \tau / 2)-\frac{2}{\kappa} \gamma C\left\langle\left[\sigma_{-}(\tau), \sigma_{+}\right]\right\rangle,
\end{aligned}
$$

where $\sigma_{ \pm}=\left(\sigma_{x} \pm i \sigma_{y}\right) / 2$ are the (single-atom) atomic raising and lowering operators.

Given that the effective atomic dynamics can be described by generalized OBE's of the form

$$
\begin{gathered}
\left\langle\dot{\sigma}_{x}\right\rangle=-\gamma_{x}\left\langle\sigma_{x}\right\rangle, \\
\left\langle\dot{\sigma}_{y}\right\rangle=-\gamma_{y}\left\langle\sigma_{y}\right\rangle+\Omega_{z}\left\langle\sigma_{z}\right\rangle, \\
\left\langle\dot{\sigma}_{z}\right\rangle=-\gamma_{z}\left\langle\sigma_{z}\right\rangle-\frac{\gamma}{2}(1+2 C)-\Omega_{y}\left\langle\sigma_{y}\right\rangle,
\end{gathered}
$$

the quantity $A_{\mathrm{p}}(\nu)$, or probe transmission, can be derived (using the quantum regression theorem to compute the required atomic correlation function) as

$$
\begin{aligned}
A_{p}(\nu)= & \frac{1}{\kappa}-\frac{\gamma C}{\kappa}\left\langle\sigma_{z}\right\rangle_{s s}\left\{\frac{1}{p^{s}}\left[\frac{\lambda_{+}^{s}+\gamma_{y}}{\lambda_{-}^{s}+i \nu}-\frac{\lambda_{-}^{s}+\gamma_{y}}{\lambda_{+}^{s}+i \nu}\right]\right. \\
& \left.-\frac{1}{\gamma_{x}-i \nu}+\frac{\Omega_{z}^{2}}{\gamma_{y} p^{s}}\left[\frac{-1}{\lambda_{+}^{s}+i \nu}+\frac{1}{\lambda_{-}^{s}+i \nu}\right]\right\},
\end{aligned}
$$

where 


$$
\left\langle\sigma_{z}\right\rangle_{s s}=-\frac{\gamma(1+2 C) \gamma_{y}}{\gamma_{z} \gamma_{y}+\Omega_{z} \Omega_{y}}
$$

and

$$
\lambda_{ \pm}^{s}=-\frac{1}{2}\left(\gamma_{y}+\gamma_{z}\right) \pm \frac{1}{2} p^{s}, \quad p^{s}=\sqrt{\left(\gamma_{y}-\gamma_{z}\right)^{2}-4 \Omega_{y} \Omega_{z}} .
$$

Of particular interest is the transmission on resonance

$$
A_{p}(\nu=0)=\frac{2}{\kappa}\left\{1+\frac{\gamma}{2} C\left\langle\sigma_{z}\right\rangle_{s s}\left[\frac{1}{\gamma_{x}}+\frac{1}{\gamma_{y}}\left(\frac{\gamma_{y} \gamma_{x}-\Omega_{z}^{2}}{\gamma_{y} \gamma_{z}+\Omega_{y} \Omega_{z}}\right)\right]\right\},
$$

which we note is purely real.

Analytical expressions for $\gamma_{x, y, z}$ and $\Omega_{y, z}$, derived for the case of finite-bandwidth squeezed light, are given in the Appendix. While these expressions are rather complicated and lengthy, we note that everything is fixed by $C, b_{ \pm}$[or $G_{+}$ via Eqs. (12) and (13)], and the strength of the drive field $\Omega$. These are all measured parameters of each instance of the experiment, so we should have a complete theory to compare to the experiment (albeit a grossly simplified one in terms of the atomic beam with the associated complications posed by the spectator atoms).

Despite the added complication, and perhaps surprisingly, the transition to narrow-band squeezing has only a small effect on the transmission spectra shown in Fig. 2, even though our squeezing bandwidth does not cover the full enhanced atomic linewidth. Rather than pursue this point further, we will now turn our attention to the phase-sensitive technique.

The quantities we focus on are formally the maximum and minimum of the phase-sensitive transmission amplitude. We define the normalized modulation amplitudes as

$$
\epsilon_{ \pm}^{p-p} \equiv \frac{A_{p}^{ \pm}(\nu=0)-A_{p}^{\mathrm{no} \mathrm{sqz}}(\nu=0)}{A_{p}^{\text {no sqz }}(\nu=0)},
$$

in which $A_{p}^{\text {no sqz }}(\nu=0)$ represents the transmission amplitude with only the coherent reference field and no squeezing, $A_{p}^{+}(\nu=0)$ is the amplitude transmission when the noisy quadrature of the squeezing ellipse is aligned with the reference field $(\phi=0)$, and $A_{p}^{-}(\nu=0)$ is the amplitude transmission when the quiet quadrature of the squeezing ellipse is aligned along the reference field $(\phi=\pi / 2)$, with reference to the definition of $\phi$ from Fig. 8. Equation (43) is a measure of the height above $\left(\epsilon^{p-p}>0\right)$ or below $\left(\epsilon^{p-p}<0\right)$ the vacuumonly level.

We must determine the behavior of the probe transmission for inputs other than squeezed light in order to identify an effect that is unique to squeezed light. Rather than perform an exhaustive search, we will take a classical squeezed field as the test case, as it is a nonquantum field with fluctuations that vary with phase (see the Appendix for how we model this case). In Figs. 14 and 15 we plot the normalized envelope of the modulation $\epsilon_{ \pm}^{p-p}$ for both quantum squeezed light and classical squeezed light as a function of OPO gain $G_{+}$for different values of the coherent field amplitude. Parameters are chosen to be similar to those of our DOPO and

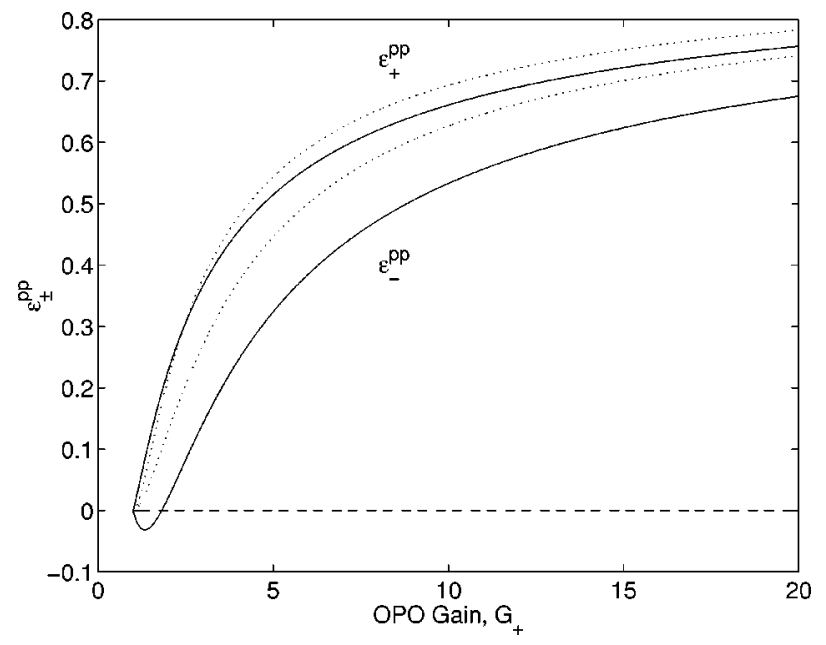

FIG. 14. Envelope of amplitude modulation $\epsilon_{ \pm}^{p-p}$ as a function of gain $G_{+}$for quantum (solid line) and classical (dotted line) squeezed light. The parameters are $\gamma_{\|}=5 \mathrm{MHz}, 2 \kappa=168 \mathrm{MHz}, C$ $=0.95, \eta=1$, and $\kappa_{s}=2.4 \gamma_{\|}$, as appropriate for the experiment. The coherent reference field amplitude is $\Omega=0.8 \gamma$.

1D atom: $\gamma_{\|}=5 \mathrm{MHz}, 2 \kappa=168 \mathrm{MHz}, C=0.95, \eta=1$, and $\kappa_{s}=2.4 \gamma_{\|}$, where $\eta$ is the efficiency of coupling of the squeezed field to the cavity. (This will be discussed in more detail later in this section.)

Figures 14 and 15 help outline the experimental strategy. The line at $\epsilon_{ \pm}^{p-p}=0$ serves to demarcate the vacuum-only level. If the envelope of the modulation dips below this level, this could be indicative of a purely nonclassical effect. We attempt to determine whether this is so by plotting $\epsilon_{ \pm}^{p-p}$ for both the quantum squeezed case and the classical squeezed case. In Fig. 14 only the quantum squeezed case dips below the vacuum level; the classical squeezing goes immediately up from zero gain. For this level of coherent reference field, the dip below zero does seem to be an indicator of a nonclassical effect. However, in Fig. 15 for a stronger reference field, both fields dip well below the (now irrelevant) vacuum-only level.

In fact, under the assumption that alterations of the atomic parameters $\gamma_{x, y, z}$ and $\Omega_{y, z}$ are small (as is evidently the case

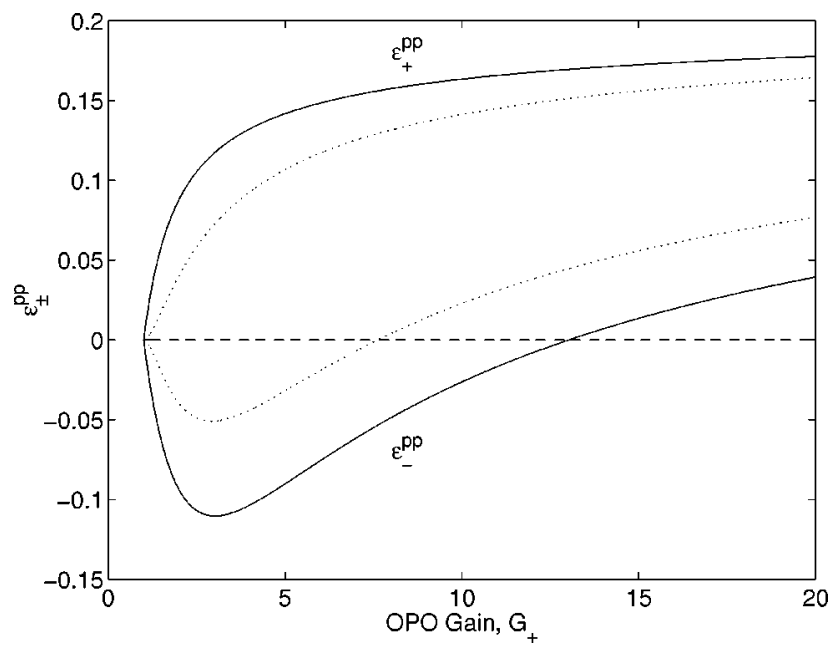

FIG. 15. Same as in Fig. 14, but with larger coherent reference field amplitude $\Omega=2 \gamma$. 
for the present experiment), it is possible to express the amplitude $A_{p}^{ \pm}(\nu=0)$ as the sum of the vacuum-only value $A_{p}^{\mathrm{nosqz}}(\nu=0)$ and a correction term that is directly proportional to the small deviations of the atomic parameters from their vacuum-only values. One is then able to identify explicitly a regime in which a negative value of the correction, i.e., a dip below the vacuum-only level, can only result from negative deviations in the atomic parameters; in particular, from reductions in one or more decay rates below their vacuum-only level, which can only occur with quantum squeezed light.

Thus there does appear to be a regime in which quantum squeezing has a unique effect, but it is a limited regime. Unfortunately, in addition, the regime is defined by the drive field strength, which is not necessarily known to sufficient absolute accuracy, and the relevant regime is also in general for low gain, where the overall modulation signal is smallest.

Finally, we note that we have tested this analytic theory against a full numerical simulation of the master equation for the combined quantum OPO plus atom-cavity system. The results are that the two approaches give reasonable agreement with one another, with the decorrelation approximation giving a slight underestimate of the size of the probe transmission modulation over the limited set of parameters tested. It is important to keep in mind, then, that while the analytic decorrelation theory provides a very convenient test for comparison with the data, that it is not exactly correct, even for an ideal scenario. The alternative of running complete numerical simulations is, however, unreasonable given the computational demands of such a task. Indeed, it is not at all clear that the effort would be worthwhile given that the experiment presents complications for which there is little hope of making direct calculations.

\section{COMPARISON OF THEORY AND DATA}

The discussion of the comparison of theory and data begins with the parameter $\eta$. All experimental evidence indicates that, simply put, the size of observed effects is just too small. An immediate guess as to why this may be the case points towards some form of loss of degree of squeezing at the site of the atom. We parametrize the overall loss by the quantity $\eta$.

To underscore this hypothesis, consider first the probe transmission spectra measurements of Sec. II D 1. By far, the most disappointing aspects of these measurements are the overall size of the effects and the relative height of the two difference spectrum peaks (those due to squeezed and thermal reservoirs). Regarding the size of the effects, from Fig. 2 we expect $\Delta_{s} \sim 0.4$ for the range of OPO gains of Fig. 7, whereas $\Delta_{s} \sim 0.1$ is measured. As for the relative heights, the prediction based on the theory of Sec. III is that the height of $\Delta_{s}$ should be approximately half as large as that of $\Delta_{t}$ (a Lorentzian that narrows without losing height will lead to a difference spectrum that dips to zero at line center), while in Fig. 7 they are almost indistinguishable. As shown in Fig. 16 , it is only as the efficiency $\eta$ of coupling of the squeezed (and thermal) field to the atom cavity is decreased that the two predictions fall atop one another (note that these curves are calculated for lower OPO gain than the data of Fig. 7).

Even with a significant effort to improve the matching of

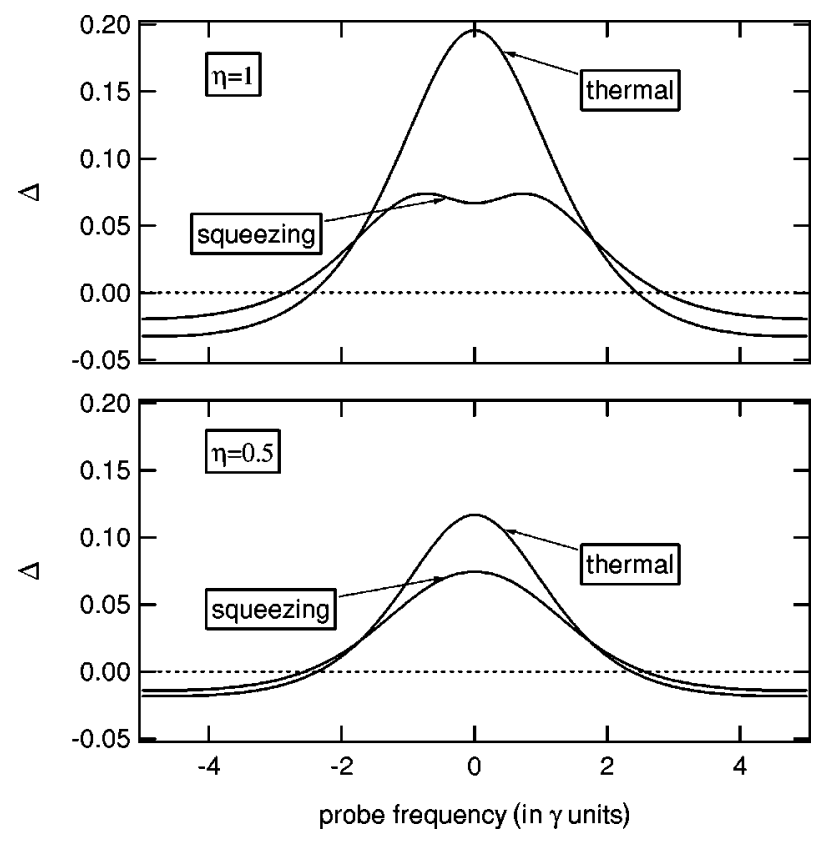

FIG. 16. As the coupling efficiency $\eta$ is decreased, the difference spectra $\Delta_{s, t}$ for squeezed and thermal excitation become increasingly difficult to distinguish.

the squeezed field to the cavity and to minimize propagation losses, the overall size of the effects never improved. To confirm that the squeezing was actually surviving the trip between laboratories, a homodyne detector was constructed immediately in front of the atom cavity and the squeezing was measured to be in agreement with that obtained just outside the OPO cavity. (In addition, the correspondence between the OPO gain and the degree of squeezing was experimentally confirmed so that a simple measure of the OPO phase-sensitive gain is sufficient to confirm the presence of squeezing.)

Let us now consider the losses in more detail. These losses come from several sources. The first is the transport efficiency $\eta_{t}=0.92$ of the squeezed light from the output of the OPO in laboratory 2 to just before mirror $M_{2}$ of the 1D atom cavity in laboratory 1 . The second contribution comes from a birefringence of the 1D atom cavity that causes the circulating polarization to be slightly different from the external polarizations, which are essentially perfect (to $1 \%$ ). This causes an effective loss in the interacting $\sigma^{+}$polarization, modeled by an $\eta_{b}=0.91$. The third loss mechanism is due to imperfect mode matching of the squeezed beam to the $1 \mathrm{D}$ atom cavity, giving $\eta_{m m}=0.95$. The fourth loss is due to actual internal cavity scattering and absorption losses at the mirror coating as quoted above in the quantity $\delta_{0}$, which leads to $\eta_{c}=0.77$. Together then, all the easily measurable loss contributions lead to a total "experimental" loss $\eta_{\text {expt }}$ $=\eta_{t} \eta_{b} \eta_{m m} \eta_{c}=0.61$. If we now also include the loss due to spectator atoms $\eta_{\text {spec }}$ as discussed in Sec. II C 2 and define the total loss $\eta_{\text {tot }}=\eta_{\text {expt }} \eta_{\text {spec }}$ we get

$$
\begin{array}{lll}
C_{\text {meas }}=1.24, & \eta_{\text {spec }}=0.41, & \eta_{\text {tot }}=0.25 ; \\
C_{\text {meas }}=0.95, & \eta_{\text {spec }}=0.49, & \eta_{\text {tot }}=0.30 ; \\
C_{\text {meas }}=0.70, & \eta_{\text {spec }}=0.57, & \eta_{\text {tot }}=0.35 ;
\end{array}
$$




$$
C_{\text {meas }}=0.55, \quad \eta_{\text {spec }}=0.63, \quad \eta_{\text {tot }}=0.38 .
$$

Unfortunately, at these levels of $\eta_{\text {tot }}$, the difference between squeezed, thermal, and classically squeezed fields is difficult to resolve. This is not surprising since the quantum field becomes more "classical" the larger the losses.

Excessive losses plague us in the same way with the phase-sensitive measurements. The effect of increased losses due to $\eta_{\mathrm{spec}}$ is to reduce in all cases the size of $\alpha_{p p}$ (roughly by a factor ranging between 2 and 4). The distinction between the cases of quantum and classical squeezing remains (but is smaller in absolute terms) in the sense that for a given $\eta$ the size of $\alpha_{p p}$ is always greater for excitation with quantum rather than with classical squeezing. Although this is true of the theory, experimentally we have no direct way of measuring $\eta_{\text {spec }}$ and hence we cannot unambiguously distinguish between the two cases since for all intents and purposes the shape of the classical and quantum curves is identical and they differ only by an ill-quantified scaling.

Despite the tremendous difficulties inherent in a comparison of theory and data for this experiment, the data do show clear trends that are qualitatively very similar to the trends of the theory. We will therefore now attempt to determine the best estimates of the various parameters that match the theory to the data. The results are shown in Fig. 17, in which the amplitude fractional modulation $\alpha_{p p}$ is plotted as a function of various experimental parameters, with best fit curves from quantum squeezing (solid curves) and classical squeezing (dashed curves) predictions overlaid, corresponding to the fit parameters of Table I. Figures 17(a) and 17(b) compare the data of Fig. 12 ( $\alpha_{p p}$ vs probe detuning) to theory and similarly Fig. 17(c) shows the data of Fig. $10\left(\alpha_{p p}\right.$ vs OPO gain $G_{+}$) and Figs. 17(e) and 17(f) the data of Fig. 11 (top). Figure 17(d) is another set of data for $\alpha_{p p}$ vs coherent reference field power.

Before we continue, we must point out what is obvious from Fig. 17: The data do not overwhelmingly and convincingly match the theory. The small size of the effect makes the SNR quite unfavorable, thereby making a comparison of data and theory difficult. Furthermore, our only hope of demonstrating a distinct quantum effect of the squeezed-lightatom interaction is with a direct comparison to the theory since we cannot in the laboratory generate a sufficient set of classical noise reservoirs for a direct empirical comparison. Nonetheless, we can explain why it is the case that the experiment was drastically more difficult than existing theories could have predicted, which in itself is noteworthy.

The focus of our efforts to match theory to data falls mainly on the parameter $C_{\text {fit }}$. While each of the six data sets considered here is a measurement of the peak-peak probe transmission amplitude modulation as a function of different parameters, we can consider all quantities except for $C$ as known, that is, the reference power, OPO gain, and detuning are all fixed at their measured values and $\eta$ is chosen to be the value expected from $\eta_{\text {tot }}$. We then let the data choose a best value of $C_{f i t}$. From Table I notice that the values of $C_{\text {fit }}$ are consistently low relative to $C_{\text {meas }}$. ( $C_{\text {fit }}^{\text {sq }}$ gives the best fit to the theory with quantum squeezed input and $C_{\text {fit }}^{\mathrm{cl}}$ for classical squeezed input.) What do we expect for $C_{\text {fit }}$ ? We anticipate that it is only the events in which a single atom is well coupled that participate in the modulation signal, yet the experiment averages the modulation signal over all time. Consider the following simplified situation. In the absence of spectator atoms a single atom pops in and out of a position in the cavity at which it is optimally coupled. (It does this on a time scale much faster than the modulation frequency.) During those times when the atom is optimally coupled, we measure a transmission amplitude modulation of size $A$. When the atom is not in the cavity (uncoupled) we observe a dc level of the empty cavity, with no modulation. If the atom is coupled to the cavity for some fraction of the time $f$, then the overall modulation signal that we measure will be an average of the optimal modulation with a signal that has no modulation. Thus the amplitude of the modulation will be reduced to a value $f A$. This situation is not all that far removed from the experiment. In reality a single near-optimally coupled atom pops in and out of the cavity mode from the sea of spectator atoms. It does this on a time scale corresponding to the transit time of atoms crossing the cavity mode $T_{0} \sim 200 \mathrm{~ns}$, which is quite fast compared to the $300-\mathrm{Hz}$ modulation frequency. If we separate the effect of the spectator atoms and lump that into the loss parameter $\eta_{\mathrm{spec}}$, then the remaining effects are due to a single atom of interest. Because we define the boundary between the domain of spectator atoms and that of the mode itself to be $g_{s_{i}}^{2} / g_{0}^{2} \sim(0.56)^{2}$ (Sec. II A 2), this single atom experiences a range of couplings from $0.3 C_{1}$ to $C_{1}$. From simulations of the atomic beam (or from analytic calculations) it is seen that the atom is strongly coupled [i.e., $g(r)>0.56 g_{0}$, Sec. II A 2] anywhere from $60 \%$ to $30 \%$ of the time (depending on the atomic flux and hence $C_{\text {meas }}$ and how one treats the cases with two or more optimally coupled atoms). What we are effectively achieving is to measure an effective value of the near-optimally coupled events, weighted over both their spatial coupling and the frequency with which they attain that coupling. Thus the values of $C_{\text {fit }}$ are in keeping with the 30-60\% occupation frequency and the $0.3 C_{1} \rightarrow C_{1}$ coupling range.

These parameters arising from the distribution of atoms in the atomic beam ( $\eta_{\text {spec }}$ and $f$, which relates $C_{\text {meas }}$ to an effective single-atom coupling strength) play a crucial role in our ability to distinguish between classical and quantum theory. For the data of Fig. 17, fits to the quantum squeezing and classical squeezing theories are of indistinguishable quality, yet yield consistently different values for $C_{\text {fit }}$ over a wide range of parameters $\left(C_{\mathrm{fit}}^{\mathrm{sq}}<C_{\mathrm{fit}}^{\mathrm{cl}}\right.$ in all cases. $)$ A more precise knowledge of $\eta_{\text {spec }}, f$, and experimental parameters $G_{+}, \bar{N}$, and $P_{\text {ref }}$ would uniquely determine the expected value of the single-atom effective coupling and the agreement (or otherwise) of $C_{\text {fit }}^{\text {sq }}$ and $C_{\text {fit }}^{\mathrm{cl}}$ with this value could then be used to distinguish the theories. For a well-parametrized atomic beam, a somewhat more rigorous approach would be to average the theoretically predicted modulation over the distribution of atomic positions in the cavity mode (estimated by simulation of the atomic beam). This averaged theory could then be directly compared with the data traces and along with accurate knowledge of our experimental parameters possibly yield a distinction between quantum and classical descriptions of the system.

In the absence of such a precise knowledge of the atomic beam, the results for $C_{\text {fit }}$ are found to be consistent with our 

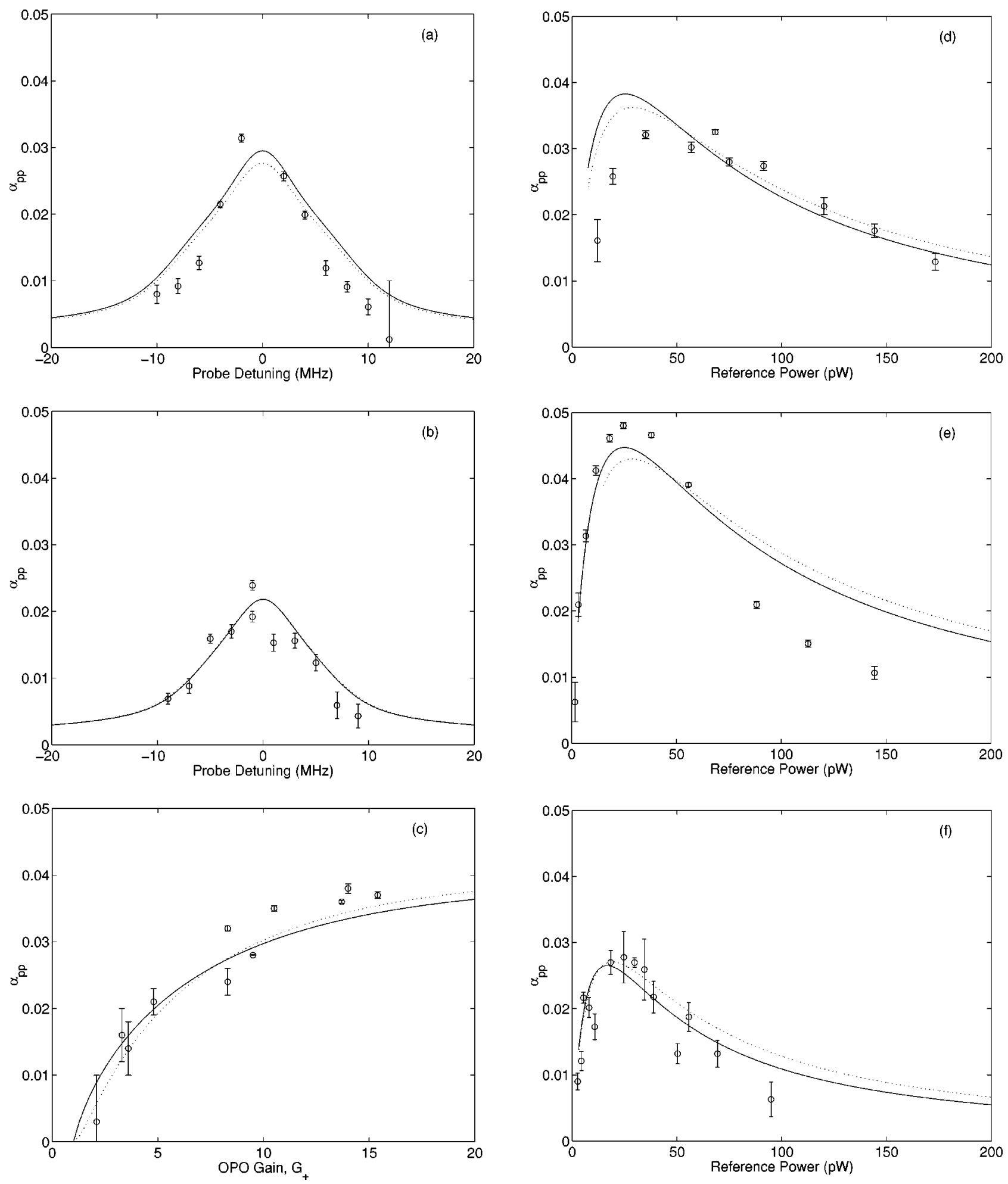

FIG. 17. Theory on data. The results are summarized in Table I. The solid curves are the best fit to the quantum squeezed prediction and the dashed curves are the best fit to the classical squeezing prediction. The quality of the fit and the measured constraints on the parameters are insufficient to distinguish between the two theories.

estimates of $\eta_{\text {spec }}$ and $f$ for either theory and are taken to be a reasonable corroboration of the following two ideas: (i) that the spectator atoms lead to an additional loss factor that greatly degrades the size of our modulation signals and (ii) that the intermittency of those atom-cavity events that give rise to the modulation signal (the well-coupled events) and their range of coupling result in a reduced effective cooperativity.

There is more to consider in the data-theory comparison. The gain is a directly measured quantity and can be simply related to the parameters of the theory, so it poses no particular difficulties. The reference power, however, does not 
TABLE I. Summary of the fitting parameters.

\begin{tabular}{lccccccc}
\hline \hline Data set & $C_{\text {meas }}$ & $C_{\text {fit }}^{\text {so }}$ & $C_{\text {fit }}^{\text {cl }}$ & $S_{c}$ & $G_{+}$ & $\eta_{\text {spec }}$ & $\eta_{\text {tot }}$ \\
\hline Fig. 17(a) & 1.24 & 0.19 & 0.20 & 33 & 14.0 & 0.44 & 0.268 \\
Fig. 17(b) & 1.24 & 0.18 & 0.21 & 33 & 6.5 & 0.44 & 0.268 \\
Fig. 17(c) & 0.95 & 0.27 & 0.33 & 27.9 & & 0.52 & 0.317 \\
Fig. 17(d) & 0.95 & 0.20 & 0.23 & 27.9 & 5.4 & 0.52 & 0.317 \\
Fig. 17(e) & 0.70 & 0.24 & 0.28 & 23.8 & 10.1 & 0.61 & 0.372 \\
Fig. 17(f) & 0.55 & 0.18 & 0.23 & 21.6 & 5.1 & 0.67 & 0.409 \\
\hline \hline
\end{tabular}

appear directly in the theory; instead the parameter $\Omega$ does. In principle, it is possible to relate the two quantities as long as all the details of the experiment are well known. Given the uncertainties of these parameters, there is a better way to attack the problem though. We have both measured and calculated "saturation" curves. The measured curve comes from blocking the output of the OPO while scanning the power of the reference field and is shown, for example, in the bottom graph of Fig. 11. The calculated version comes from the theory by simply "shutting off" the squeezing and scanning the power of $\Omega$. By fitting the two with a free scaling parameter $S_{c}$ the two quantities can be related and picowatts of reference power can be converted to values of $\Omega^{2}$, with the results shown in Table I. Of course this method suffers from the same difficulties as discussed above, but is adequate for the purpose at hand. This method accounts for factors that would otherwise be difficult to take into account, such as the difference in saturation between one localized atom with cooperativity $C$ and a sample of atoms with $\bar{N}=1$ and $\bar{N} C_{1}=C$.

\section{CONCLUSION}

Given the complexity and difficulty of this experiment, it is remarkable that the data follow a trend faithful to a simplified (yet still complicated) theory. We have observed modification in the response of our atom-cavity system when a squeezed vacuum is injected into the cavity. In particular, the transmission to a weak probe beam is dependent on the phase of the squeezed field relative to a coherent saturating field, resulting in a modulated probe transmission as the phase of the squeezing is scanned. We have mapped out the amplitude of this modulation as a function of our various experimental parameters, OPO gain, probe detuning, coherent saturating field strength, and atom number, fitting theory curves to these data to infer the coupling strength.

It is unfortunate that we are not, however, able to make any definitive statement that we are observing manifestations of the nonclassical interaction between atoms and squeezed light, a result that we attribute to unforeseen additional loss mechanisms arising from our thermal atomic beam as a source of atoms. This experiment, along with Ref. [99], points to the unsuitability of this type of thermal source for measurement of single-atom quantum effects and to the desirability of developing methods for the real-time interrogation of true single atoms, such as those currently being developed in our group [100,101]. Despite the lack of an unequivocal observation of nonclassical effects, there are nonetheless unquestionably some interesting results from the experiment pertaining to issues that have never before seen the harsh light of the laboratory.

\section{ACKNOWLEDGMENTS}

We appreciate the assistance of Professor Eugene Polzik and Dr. Robert J. Thompson in the early stages of the experiment. This work was supported by the Division of Chemical Sciences, Office of Basic Energy Sciences, Office of Energy Research, U.S. Department of Energy.

\section{APPENDIX: FURTHER THEORETICAL DETAILS}

In this appendix we outline the theoretical approach we have taken to deriving expressions for the parameters $\gamma_{x, y, z}$ and $\Omega_{y, z}$ appearing in the single-atom optical Bloch equations. Having adiabatically eliminated the cavity mode and following, for example, Refs. [22,29], one can derive the stochastic OBE's

$$
\begin{gathered}
\dot{\sigma}_{x}=-\left[(\gamma / 2)\left(1+2 C_{1}\right)+\gamma_{\mathrm{tr}}\right] \sigma_{x}+\alpha_{X}(t) \sigma_{z}, \\
\dot{\sigma}_{y}=-\left[(\gamma / 2)\left(1+2 C_{1}\right)+\gamma_{\mathrm{tr}}\right] \sigma_{y}+\Omega \sigma_{z}+\alpha_{Y}(t) \sigma_{z} \\
\dot{\sigma}_{z}=-\gamma\left(1+2 C_{1}\right)-\gamma\left(1+2 C_{1}\right) \sigma_{z}-\Omega \sigma_{y}-\alpha_{X}(t) \sigma_{x} \\
-\alpha_{Y}(t) \sigma_{y},
\end{gathered}
$$

where $\alpha_{X}(t)$ and $\alpha_{Y}(t)$ are noise terms modeling the effects of squeezing from the OPO output on the effective cavitymodified vacuum field experienced by the cavity-confined atom. The statistics of these noise sources are defined by the moments

$$
\begin{gathered}
\left\langle\alpha_{X}(t)\right\rangle=\left\langle\alpha_{Y}(t)\right\rangle=0 \\
\left\langle\alpha_{X}(t) \alpha_{X}\right\rangle= \pm 2 \eta \gamma C_{1} \frac{b_{+}^{2}-b_{-}^{2}}{2 b_{\mp}} e^{-b_{\mp} t} \\
\equiv 2 \eta \gamma C_{1}(N \pm M) b_{\mp} e^{-b_{\mp} t} \\
\left\langle\alpha_{Y}(t) \alpha_{Y}\right\rangle=\mp 2 \eta \gamma C_{1} \frac{b_{+}^{2}-b_{-}^{2}}{2 b_{ \pm}} e^{-b_{ \pm} t} \\
\equiv 2 \eta \gamma C_{1}(N \mp M) b_{ \pm} e^{-b_{ \pm} t}
\end{gathered}
$$

where we have used the notation $b_{ \pm}=\left(\kappa_{s} / 2\right) \pm \epsilon$ with $\kappa_{s}$ the OPO cavity linewidth $\left[x=\epsilon /\left(\kappa_{s} / 2\right)\right]$ and \pm corresponds to the two choices of relative phase between coherent and squeezed fields $\phi=0$ and $\phi=\pi / 2$. The parameter $\eta$ is the 
efficiency of coupling of the squeezed field to the cavity mode. We note finally that the additional parameter $\gamma_{\text {tr }}$ appearing in the OBE's has been added to model the effects of transit broadening in the experiment.

We proceed by formally integrating two of the above equations and substituting the resultant expressions into the third equation; this is repeated for each equation. Averaging over the noise, we make a so-called decorrelation approximation, whereby, e.g., $\left\langle\alpha_{X}(t) \alpha_{X}\left(t^{\prime}\right) \sigma_{X}\left(t^{\prime}\right)\right\rangle$ $\rightarrow\left\langle\alpha_{X}(t) \alpha_{X}\left(t^{\prime}\right)\right\rangle\left\langle\sigma_{x}\left(t^{\prime}\right)\right\rangle$. Noting also that for the choices of phase $\phi=0$ and $\phi=\pi / 2$ one has $\left\langle\alpha_{X}(t) \alpha_{Y}\left(t^{\prime}\right)\right\rangle=0$, the following equations result:

$$
\begin{aligned}
& \frac{\partial}{\partial t}\left\langle\sigma_{x}\right\rangle=-\left[(\gamma / 2)\left(1+2 C_{1}\right)+\gamma_{\mathrm{tr}}\right]\left\langle\sigma_{x}\right\rangle-\frac{1}{p} \int_{0}^{t} d t^{\prime}\left\{\left[\lambda_{+}+\frac{\gamma}{2}\left(1+2 C_{1}\right)+\gamma_{\mathrm{tr}}\right] e^{\lambda_{+}\left(t-t^{\prime}\right)}\right. \\
& \left.-\left[\lambda_{-}+\frac{\gamma}{2}\left(1+2 C_{1}\right)+\gamma_{\mathrm{tr}}\right] e^{\lambda_{-}\left(t-t^{\prime}\right)}\right\}\left\langle\alpha_{X}(t) \alpha_{X}\left(t^{\prime}\right)\right\rangle\left\langle\sigma_{x}\left(t^{\prime}\right)\right\rangle, \\
& \frac{\partial}{\partial t}\left\langle\sigma_{y}\right\rangle=-\left[(\gamma / 2)\left(1+2 C_{1}\right)+\gamma_{\mathrm{tr}}\right]\left\langle\sigma_{y}\right\rangle+\Omega\left\langle\sigma_{z}\right\rangle-\frac{1}{p} \int_{0}^{t} d t^{\prime}\left\{\left[\lambda_{+}+\frac{\gamma}{2}\left(1+2 C_{1}\right)+\gamma_{\mathrm{tr}}\right] e^{\lambda_{+}\left(t-t^{\prime}\right)}\right. \\
& \left.-\left[\lambda_{-}+\frac{\gamma}{2}\left(1+2 C_{1}\right)+\gamma_{\mathrm{tr}}\right] e^{\lambda_{-}\left(t-t^{\prime}\right)}\right\}\left\langle\alpha_{Y}(t) \alpha_{Y}\left(t^{\prime}\right)\right\rangle\left\langle\sigma_{y}\left(t^{\prime}\right)\right\rangle-\frac{\Omega}{p} \int_{0}^{t} d t^{\prime}\left[e^{\lambda_{+}\left(t-t^{\prime}\right)}-e^{\lambda_{-}\left(t-t^{\prime}\right)}\right] \\
& \times\left\langle\alpha_{Y}(t) \alpha_{Y}\left(t^{\prime}\right)\right\rangle\left\langle\sigma_{z}\left(t^{\prime}\right)\right\rangle, \\
& \frac{\partial}{\partial t}\left\langle\sigma_{z}\right\rangle=-\gamma\left(1+2 C_{1}\right)-\gamma\left(1+2 C_{1}\right)\left\langle\sigma_{z}\right\rangle-\Omega\left\langle\sigma_{y}\right\rangle-\frac{1}{p} \int_{0}^{t} d t^{\prime}\left\{\left[\lambda_{+}+\frac{\gamma}{2}\left(1+2 C_{1}\right)+\gamma_{\mathrm{tr}}\right] e^{\lambda_{-}\left(t-t^{\prime}\right)}\right. \\
& \left.-\left[\lambda_{-}+\frac{\gamma}{2}\left(1+2 C_{1}\right)+\gamma_{\mathrm{tr}}\right] e^{\lambda_{+}\left(t-t^{\prime}\right)}\right\}\left\langle\alpha_{Y}(t) \alpha_{Y}\left(t^{\prime}\right)\right\rangle\left\langle\sigma_{z}\left(t^{\prime}\right)\right\rangle-\int_{0}^{t} d t^{\prime} e^{-\left[(\gamma / 2)\left(1+2 C_{1}\right)+\gamma_{\mathrm{tr}}\right]\left(t-t^{\prime}\right)}\left\langle\alpha_{X}(t) \alpha_{X}\left(t^{\prime}\right)\right\rangle\left\langle\sigma_{z}\left(t^{\prime}\right)\right\rangle \\
& +\frac{\Omega}{p} \int_{0}^{t} d t^{\prime}\left[e^{\lambda_{+}\left(t-t^{\prime}\right)}-e^{\lambda_{-}\left(t-t^{\prime}\right)}\right]\left\langle\alpha_{Y}(t) \alpha_{Y}\left(t^{\prime}\right)\right\rangle\left\langle\sigma_{y}\left(t^{\prime}\right)\right\rangle
\end{aligned}
$$

where

$$
\lambda_{ \pm}=-\frac{3}{4} \gamma\left(1+2 C_{1}\right)-\frac{1}{2} \gamma_{\mathrm{tr}} \pm \frac{1}{2} p, \quad p=\sqrt{\left[(\gamma / 2)\left(1+2 C_{1}\right)-\gamma_{\mathrm{tr}}\right]^{2}-4 \Omega^{2}}
$$

Assuming reasonably fast decay of the exponentials, one can set the upper integral limits to infinity. If we also assume that $\Omega$ is sufficiently large that $p$ is complex and perhaps also of the order of, or larger than, the decay rates in the exponentials, then reasonable substitutions for $\left\langle\sigma_{y}\left(t^{\prime}\right)\right\rangle$ and $\left\langle\sigma_{z}\left(t^{\prime}\right)\right\rangle$ in the integrals are

$$
\begin{aligned}
& \left\langle\sigma_{y}\left(t^{\prime}\right)\right\rangle \simeq \cos \left[(p / 2 i)\left(t-t^{\prime}\right)\right]\left\langle\sigma_{y}(t)\right\rangle-\sin \left[(p / 2 i)\left(t-t^{\prime}\right)\right]\left\langle\sigma_{z}(t)\right\rangle, \\
& \left\langle\sigma_{z}\left(t^{\prime}\right)\right\rangle \simeq \cos \left[(p / 2 i)\left(t-t^{\prime}\right)\right]\left\langle\sigma_{z}(t)\right\rangle+\sin \left[(p / 2 i)\left(t-t^{\prime}\right)\right]\left\langle\sigma_{y}(t)\right\rangle .
\end{aligned}
$$

In this way, the integrodifferential equations above are reduced to differential equations of the form (36), with the various coefficients to be determined by evaluating the various integrals.

In particular, these coefficients are (having used the substitutions above)

$$
\begin{aligned}
& \gamma_{x}=\frac{1}{2} \gamma\left(1+2 C_{1}\right)+\gamma_{\mathrm{tr}}+2 \gamma C_{1} \eta(N \mp M) \frac{1}{p} b_{ \pm}\left[-\frac{\lambda_{+}+(\gamma / 2)\left(1+2 C_{1}\right)+\gamma_{\mathrm{tr}}}{\lambda_{+}-b_{ \pm}} \frac{\lambda_{-}+(\gamma / 2)\left(1+2 C_{1}\right)+\gamma_{\mathrm{tr}}}{\lambda_{-}}-b_{ \pm}\right. \\
\gamma_{y}= & \frac{1}{2} \gamma\left(1+2 C_{1}\right)+\gamma_{\mathrm{tr}}+\gamma C_{1} \eta(N \pm M) \frac{\Omega}{i p} b_{\mp}\left[-\frac{1}{\lambda_{+}-b_{\mp}+p / 2}+\frac{1}{\lambda_{+}-b_{\mp}-p / 2}+\frac{1}{\lambda_{-}-b_{\mp}+p / 2}-\frac{1}{\lambda_{-}-b_{\mp}-p / 2}\right] \\
& +\gamma C_{1} \eta(N \pm M) \frac{1}{p} b_{\mp}\left\{-\left[\lambda_{+}+(\gamma / 2)\left(1+2 C_{1}\right)+\gamma_{\mathrm{tr}}\right]\left(\frac{1}{\lambda_{+}-b_{\mp}+p / 2}+\frac{1}{\lambda_{+}-b_{\mp}-p / 2}\right)+\left[\lambda_{-}+(\gamma / 2)\left(1+2 C_{1}\right)+\gamma_{\mathrm{tr}}\right]\right. \\
& \left.\times\left(\frac{1}{\lambda_{-}-b_{\mp}+p / 2}+\frac{1}{\lambda_{-}-b_{\mp}-p / 2}\right)\right\}
\end{aligned}
$$




$$
\begin{aligned}
& \gamma_{z}=\gamma\left(1+2 C_{1}\right)+\gamma C_{1} \eta(N \pm M) \frac{1}{p} b_{\mp}\left\{-\left[\lambda_{+}+(\gamma / 2)\left(1+2 C_{1}\right)+\gamma_{\mathrm{tr}}\right]\left(\frac{1}{\lambda_{-}-b_{\mp}+p / 2}+\frac{1}{\lambda_{-}-b_{\mp}-p / 2}\right)\right. \\
& \left.+\left[\lambda_{-}+(\gamma / 2)\left(1+2 C_{1}\right)+\gamma_{\mathrm{tr}}\right]\left(\frac{1}{\lambda_{+}-b_{\mp}+p / 2}+\frac{1}{\lambda_{+}-b_{\mp}-p / 2}\right)\right\} \\
& -\gamma C_{1} \eta(N \pm M) \frac{\Omega}{i p} b_{\mp}\left(\frac{1}{\lambda_{+}-b_{\mp}+p / 2}-\frac{1}{\lambda_{+}-b_{\mp}-p / 2}-\frac{1}{\lambda_{-}-b_{\mp}+p / 2}+\frac{1}{\lambda_{-}-b_{\mp}-p / 2}\right) \\
& +\gamma C_{1} \eta(N \mp M) b_{ \pm}\left\{\frac{1}{\left[(\gamma / 2)\left(1+2 C_{1}\right)+\gamma_{\mathrm{tr}}\right]+b_{ \pm}-(p / 2)}+\frac{1}{\left[(\gamma / 2)\left(1+2 C_{1}\right)+\gamma_{\mathrm{tr}}\right]+b_{ \pm}+(p / 2)}\right\}, \\
& \Omega_{z}=\Omega-\gamma C_{1} \eta(N \pm M) \frac{\Omega}{p} b_{\mp}\left[-\frac{1}{\lambda_{+}-b_{\mp}+p / 2}-\frac{1}{\lambda_{+}-b_{\mp}-p / 2}+\frac{1}{\lambda_{-}-b_{\mp}+p / 2}+\frac{1}{\lambda_{-}-b_{\mp}-p / 2}\right] \\
& +\gamma C_{1} \eta(N \pm M) \frac{1}{i p} b_{\mp}\left\{-\left[\lambda_{+}+(\gamma / 2)\left(1+2 C_{1}\right)+\gamma_{\mathrm{tr}}\right]\left(\frac{1}{\lambda_{+}-b_{\mp}+p / 2}-\frac{1}{\lambda_{+}-b_{\mp}-p / 2}\right)\right. \\
& \left.+\left[\lambda_{-}+(\gamma / 2)\left(1+2 C_{1}\right)+\gamma_{\mathrm{tr}}\right]\left(\frac{1}{\lambda_{-}-b_{\mp}+p / 2}-\frac{1}{\lambda_{-}-b_{\mp}-p / 2}\right)\right\} \\
& \Omega_{y}=\Omega+\gamma C_{1} \eta(N \pm M) \frac{1}{i p} b_{\mp}\left\{-\left[\lambda_{+}+(\gamma / 2)\left(1+2 C_{1}\right)+\gamma_{\mathrm{tr}}\right]\left(\frac{1}{\lambda_{-}-b_{\mp}+p / 2}-\frac{1}{\lambda_{-}-b_{\mp}-p / 2}\right)\right. \\
& \left.+\left[\lambda_{-}+(\gamma / 2)\left(1+2 C_{1}\right)+\gamma_{\mathrm{tr}}\right]\left(\frac{1}{\lambda_{+}-b_{\mp}+p / 2}-\frac{1}{\lambda_{+}-b_{\mp}-p / 2}\right)\right\} \\
& +\gamma C_{1} \eta(N \pm M) \frac{\Omega}{p} b_{\mp}\left(\frac{1}{\lambda_{+}-b_{\mp}+p / 2}+\frac{1}{\lambda_{+}-b_{\mp}-p / 2}-\frac{1}{\lambda_{-}-b_{\mp}+p / 2}-\frac{1}{\lambda_{-}-b_{\mp}-p / 2}\right) \\
& -\gamma C_{1} \eta(N \mp M) i b_{ \pm}\left\{\frac{1}{\left[(\gamma / 2)\left(1+2 C_{1}\right)+\gamma_{\mathrm{tr}}\right]+b_{ \pm}-(p / 2)}-\frac{1}{\left[(\gamma / 2)\left(1+2 C_{1}\right)+\gamma_{\mathrm{tr}}\right]+b_{ \pm}+(p / 2)}\right\} \text {. }
\end{aligned}
$$

These expressions can be further manipulated to eliminate some factors, but the resulting forms are probably not much more enlightening than those above. For the case in which $\Omega$ is small, the substitutions of Eq. (A11) are best replaced simply with $\left\langle\sigma_{y}\left(t^{\prime}\right)\right\rangle \simeq\left\langle\sigma_{y}(t)\right\rangle$ and $\left\langle\sigma_{z}\left(t^{\prime}\right)\right\rangle \simeq\left\langle\sigma_{z}(t)\right\rangle$, which leads to a considerable simplification of the forms for the coefficients. Finally, we note that to model the effect of classical squeezing with a finite bandwidth (for comparison with the quantum squeezing case), we simply set $\alpha_{Y}(t)=0\left[\alpha_{X}(t)=0\right]$ for $\phi=0(\phi=\pi / 2)$ in the equations above.

[1] C. W. Gardiner, Phys. Rev. Lett. 56, 1917 (1986).

[2] G. J. Milburn, Opt. Acta 31, 671 (1984).

[3] G. J. Milburn, Phys. Rev. A 34, 4882 (1986).

[4] N. P. Georgiades et al., Phys. Rev. Lett. 75, 3426 (1995).

[5] Q. A. Turchette, R. J. Thompson, and H. J. Kimble, Appl. Phys. B: Lasers Opt. 60, S1 (1995).

[6] D. J. Heinzen, J. L. Childs, J. Thomas, and M. S. Feld, Phys. Rev. Lett. 58, 1320 (1987).

[7] S. E. Morin, C. C. Yu, and T. W. Mossberg, Phys. Rev. Lett. 73, 1489 (1994).

[8] C. W. Gardiner and A. S. Parkins, Phys. Rev. A 50, 1792 (1994).

[9] R. Dum, A. S. Parkins, P. Zoller, and C. W. Gardiner, Phys. Rev. A 46, 4382 (1992).

[10] J. I. Cirac and L. L. Sanchezsoto, Phys. Rev. A 40, 3743 (1989).

[11] M. L. Zou and G. C. Guo, J. Phys. B 22, 2205 (1989).

[12] C. W. Gardiner, A. S. Parkins, and M. J. Collett, J. Opt. Soc. Am. B 4, 1683 (1987).
[13] S. H. An, M. Sargent, and D. F. Walls, Opt. Commun. 67, 373 (1988).

[14] H. Ritsch and P. Zoller, Opt. Commun. 64, 523 (1987).

[15] P. Zhou, Z. Ficek, and S. Swain, J. Opt. Soc. Am. B 13, 768 (1996).

[16] Z. Ficek and M. R. B. Wahiddin, Quantum Semiclassic. Opt. 7, 41 (1995).

[17] Z. Ficek, J. Mod. Opt. 40, 2333 (1993).

[18] P. Zhou and S. Swain, Quantum Semiclassic. Opt. 8, 959 (1996).

[19] P. R. Rice and C. A. Baird, Phys. Rev. A 53, 3633 (1996).

[20] S. Smart and S. Swain, Phys. Rev. A 45, 6857 (192).

[21] H. Ritsch and P. Zoller, Phys. Rev. Lett. 61, 1097 (1988).

[22] A. S. Parkins and C. W. Gardiner, Phys. Rev. A 37, 3867 (1988).

[23] M. Bosticky, Z. Ficek, and B. J. Dalton, Phys. Rev. A 53, 4439 (1996).

[24] R. Vyas and S. Singh, Phys. Rev. A 45, 8095 (1992). 
[25] H. J. Carmichael, A. S. Lane, and D. F. Walls, J. Mod. Opt. 34, 821 (1987).

[26] H. J. Carmichael, A. S. Lane, and D. F. Walls, Phys. Rev. Lett. 58, 2539 (1987).

[27] A. S. Parkins, Phys. Rev. A 42, 6873 (1990).

[28] Z. Ficek and B. C. Sanders, J. Phys. B 27, 809 (1994).

[29] A. S. Parkins, Phys. Rev. A 42, 4352 (1990).

[30] S. Swain and P. Zhou, Opt. Commun. 123, 310 (1996).

[31] S. Swain and P. Zhou, Phys. Rev. A 52, 4845 (1995).

[32] W. S. Smyth and S. Swain, Opt. Commun. 112, 91 (1994).

[33] S. Swain, Phys. Rev. Lett. 73, 1493 (1994).

[34] S. Smart and S. Swain, Phys. Rev. A 48, R48 (1993).

[35] S. Smart and S. Swain, Quantum Opt. 5, 75 (1993).

[36] S. Smart and S. Swain, Phys. Rev. A 45, 6863 (1992).

[37] C. M. Savage and D. F. Walls, Phys. Rev. Lett. 57, 2164 (1986).

[38] A. S. Parkins, Phys. Rev. A 53, 2893 (1996).

[39] A. S. Parkins and C. W. Gardiner, Phys. Rev. A 40, 2534 (1989).

[40] M. V. Satyanarayana, P. Rice, R. Vyas, and H. J. Carmichael, J. Opt. Soc. Am. B 6, 228 (1989).

[41] M. A. M. Marte, H. Ritsch, and D. F. Walls, Phys. Rev. A 38, 3577 (1988).

[42] M. A. M. Marte, H. Ritsch, and D. F. Walls, Phys. Rev. Lett. 61, 1093 (1988).

[43] P. R. Rice et al., Phys. Rev. A 50, 4176 (1994).

[44] C. Ginzel, J. Geabanacloche, and A. Schenzle, Phys. Rev. A 42, 4164 (1990).

[45] C. Ginzel, R. Schack, and A. Schenzle, J. Opt. Soc. Am. B 8, 1704 (1991).

[46] C. Ginzel, J. G. Geabanacloche, and A. Schenzle, Acta Phys. Pol. A 78, 123 (1990).

[47] M. A. M. Marte and D. F. Walls, Phys. Rev. A 37, 1235 (1988).

[48] Z. Ficek, W. S. Smyth, and S. Swain, Opt. Commun. 110, 555 (1994).

[49] Z. Ficek, W. S. Smyth, and S. Swain, Phys. Rev. A 52, 4126 (1995).

[50] Z. Ficek and B. J. Dalton, Opt. Commun. 102, 231 (1993).

[51] J. I. Cirac and L. L. Sanchezsoto, Phys. Rev. A 44, 1948 (1991).

[52] T. A. B. Kennedy and D. F. Walls, Phys. Rev. A 42, 3051 (1990).

[53] A. S. Parkins and C. W. Gardiner, Phys. Rev. A 40, 3796 (1989).

[54] J. Geabanacloche, R. R. Schlicher, and M. S. Zubairy, Phys. Rev. A 38, 3514 (1988).

[55] C. Cabrillo and S. Swain, Phys. Rev. Lett. 77, 478 (1996).

[56] W. S. Smyth and S. Swain, Phys. Rev. A 53, 2846 (1996).

[57] Y. Benaryeh, C. A. Miller, H. Risken, and W. Schleich, Opt. Commun. 90, 259 (1992).

[58] P. R. Rice and L. M. Pedrotti, J. Opt. Soc. Am. B 9, 2008 (1992)

[59] J. I. Cirac, Phys. Rev. A 46, 4354 (1992).

[60] A. S. Parkins, P. Zoller, and H. J. Carmichael, Phys. Rev. A 48, 758 (1993).

[61] R. Graham, D. F. Walls, and W. P. Zhang, Phys. Rev. A 44, 7777 (1991).

[62] J. I. Cirac and P. Zoller, Phys. Rev. A 47, 2191 (1993).

[63] Y. Shevy, Phys. Rev. Lett. 64, 2905 (1990).
[64] Y. Shevy, B. Crosignani, and A. Yariv, Phys. Rev. A 46, 1421 (1992).

[65] J. Geabanacloche, Phys. Rev. Lett. 62, 1603 (1989).

[66] J. Javanainen and P. L. Gould, Phys. Rev. A 41, 5088 (1990).

[67] Z. Ficek and P. D. Drummond, Europhys. Lett. 24, 455 (1993).

[68] M. R. Ferguson, Z. Ficek, and B. J. Dalton, Phys. Rev. A 54, 2379 (1996).

[69] M. R. Ferguson, Z. Ficek, and B. J. Dalton, J. Mod. Opt. 42, 679 (1995).

[70] Z. Ficek and P. D. Drummond, Phys. Rev. A 43, 6247 (1991).

[71] Z. Ficek and P. D. Drummond, Phys. Rev. A 43, 6258 (1991).

[72] P. Zhou and S. Swain, Phys. Rev. A 54, 2455 (1996).

[73] S. Smart and S. Swain, J. Mod. Opt. 40, 1939 (1993).

[74] S. Smart and S. Swain, J. Mod. Opt. 41, 1055 (1994).

[75] S. Smart and S. Swain, Opt. Commun. 99, 369 (1993).

[76] S. Smart and S. Swain, Quantum Opt. 4, 281 (1992).

[77] P. Galatola, L. A. Lugiato, M. G. Porreca, and P. Tombesi, Opt. Commun. 81, 175 (1991).

[78] P. Galatola and L. A. Lugiato, IOP Conference Proceedings No. 115 (Institute of Physics and Physical Society, Bristol, 1991), p. 65.

[79] Z. M. Zhang, L. Xu, and J. L. Chai, Phys. Lett. A 151, 65 (1990).

[80] G. S. Agarwal and R. R. Puri, Phys. Rev. A 41, 3782 (1990).

[81] Z. Ficek, Opt. Commun. 88, 494 (1992).

[82] Z. Ficek, Opt. Commun. 82, 130 (1991).

[83] Z. Ficek, Phys. Rev. A 44, 7759 (1991).

[84] Z. Ficek, Phys. Rev. A 42, 611 (1990).

[85] A. S. Parkins, in Modern Nonlinear Optics, Part 2, Vol. LXXXV of Advances in Chemical Physics, edited by M. W. Evans and S. Kielich (Wiley, New York, 1993), pp. 607666.

[86] H. J. Carmichael, Phys. Rev. Lett. 70, 2273 (1993).

[87] C. W. Gardiner, Phys. Rev. Lett. 70, 2269 (1993).

[88] P. R. Rice and H. J. Carmichael, IEEE J. Quantum Electron. 24, 1351 (1988)

[89] L. A. Lugiato, in Progress in Optics, edited by E. Wolf (Elsevier Science, Amsterdam, 1984), Vol. 21, p. 71.

[90] Q. A. Turchette et al., Phys. Rev. Lett. 75, 4710 (1995).

[91] M. J. Collett and R. Louden, J. Opt. Soc. Am. B 4, 1525 (1987).

[92] N. Ph. Georgiades, Ph.D. thesis, California Institute of Technology, 1998 (unpublished).

[93] H. J. Kimble, in Fundamental Systems in Quantum Optics, edited by J. Dalibard, J. M. Raimond, and J. Zinn-Justin (North-Holland, Amsterdam, 1992), pp. 545-674.

[94] C. W. Gardiner, Quantum Noise (Springer-Verlag, Berlin, 1991).

[95] E. S. Polzik, J. Carri, and H. J. Kimble, Appl. Phys. B: Photophys. Laser Chem. 55, 279 (1992).

[96] E. S. Polzik, J. Carri, and H. J. Kimble, Phys. Rev. Lett. 68, 3020 (1992).

[97] E. S. Polzik and H. J. Kimble, Opt. Lett. 16, 1400 (1991).

[98] R. J. Thompson, G. Rempe, and H. J. Kimble, Phys. Rev. Lett. 68, 1132 (1992).

[99] R. J. Thompson, Q. A. Turchette, O. Carnal, and H. J. Kimble, Phys. Rev. A 57, 3084 (1998). 
[100] H. Mabuchi, Q. A. Turchette, M. S. Chapman, and H. J. Kimble, Opt. Lett. 21, 1393 (1996).

[101] C. J. Hood, M. S. Chapman, T. W. Lynn, and H. J. Kimble, Phys. Rev. Lett. 80, 4157 (1998).

[102] Q. A. Turchette, Ph.D. thesis, California Institute of Technology, 1997 (unpublished).
[103] N. P. Georgiades et al., IQEC Technical Digest (Optical Society of America, Washington, D.C., 1994), Vol. 9, p. 222.

[104] H. J. Kimble et al., in Coherence and Quantum Optics VII, edited by J. Eberly, L. Mandel, and E. Wolf (Plenum, New York, 1996), p. 203. 